TRANSACTIONS OF THE

AMERICAN MATHEMATICAL SOCIETY

Volume 363, Number 8, August 2011, Pages 4309-4336

S 0002-9947(2011)05256-6

Article electronically published on March 1, 2011

\title{
ALGEBRAS WITH CYCLE-FINITE GALOIS COVERINGS
}

\author{
JOSÉ A. DE LA PEÑA AND ANDRZEJ SKOWROŃSKI
}

\begin{abstract}
We prove that the finite dimensional algebras over an algebraically closed field which admit cycle-finite Galois coverings with torsion-free Galois groups are of tame representation type, and derive some consequences.
\end{abstract}

\section{INTRODUCTION}

Throughout the article by an algebra we mean a finite dimensional associative $K$-algebra with an identity over a fixed algebraically closed field $K$. By a module over an algebra $A$ we mean a right $A$-module of finite dimension over $K$, if not specified otherwise.

From Drozd's remarkable Tame and Wild Theorem Dro (see also [CB1]) the class of algebras may be divided into two disjoint classes. One class consists of the tame algebras for which the indecomposable modules occur, in each dimension $d$, in a finite number of discrete and a finite number of one-parameter families. The second class is formed by the wild algebras whose representation theory comprises the representation theories of all finite dimensional algebras over $K$ (see [SS2, Chapter XIX]). Hence, a classification of the finite dimensional modules is only feasible for tame algebras. A distinguished class of tame algebras is formed by the algebras of finite type, having only finitely many isoclasses of indecomposable modules, for which the representation theory is rather well understood (see BGRS, Bo2, BoG, BrG]). On the other hand, the representation theory of arbitrary tame algebras is still only emerging. At present the most accessible seem to be the (tame) algebras of polynomial growth, for which there exists an integer $m$ such that the number of one-parameter families of indecomposable modules is bounded, in each dimension $d$, by $d^{m}$. This class of algebras has been the subject of intensive research over the last 30 years.

The main methods of the representation theory of algebras have been developed best for the triangular algebras (the Gabriel quiver has no oriented cycles), having finite global dimension (see ASS, Ri], SS1, SS2]). In order to deal with arbitrary algebras, covering techniques were introduced and developed at the beginning of the 1980s (see BoG, DS1, DS3, Ga]). Frequently, an algebra $A$ admits a Galois covering $R \rightarrow R / G=A$, where $R$ is a triangular locally bounded category and

Received by the editors June 25, 2009 and, in revised form, November 19, 2009.

2010 Mathematics Subject Classification. Primary 16G60, 16 G70.

Key words and phrases. Tame algebras, Galois coverings, cycles of modules.

Both authors acknowledge support from the Consejo Nacional de Ciencia y Technologia of Mexico.

The second author has also been supported by the research grant no. N N201 269135 of the Polish Ministry of Science and Higher Education. 
$G$ is a torsion-free group acting freely on the objects of $R$, which allows us to reduce the representation theory of $A$ to that for the corresponding algebras of finite global dimension inside $R$. Moreover, Geiss proved in Ge (see also CB3]) that if an algebra $A$ admits a tame degeneration (in the variety of algebras of a fixed dimension), then $A$ is tame. Hence, a convenient way to determine whether a given algebra $A$ is tame consists in finding a suitable tame degeneration of $A$. This was successfully applied in CB3] to prove the tameness of arbitrary biserial algebras and in BPS to show that a strongly simply connected algebra is tame if and only if its Tits quadratic form is weakly nonnegative. It is expected that every algebra $\Lambda$ of polynomial growth is Morita equivalent to an algebra $A$ (basic algebra of $\Lambda$ ) which admits a canonical degeneration $\bar{A}$ (standard form of $A$ ) of polynomial growth, having a triangular Galois covering $R \rightarrow R / G=\bar{A}$ with a torsion-free group $G$, and such that the representation theories of $A$ and $\bar{A}$ are very close. This is the case for all algebras of finite type (see [BGRS, [Bo2, [BrG]) and all self-injective algebras of polynomial growth (see [Sk1], Sk11]).

A prominent role in the representation theory of algebras is played by cycles of modules, more generally cycles of complexes of modules (see [AS3], [Sk5], [Sk7]). A cycle in the category $\bmod A$ of finite dimensional modules over an algebra $A$ is a sequence

$$
X=X_{0} \stackrel{f_{1}}{\longrightarrow} X_{1} \stackrel{f_{2}}{\longrightarrow} \ldots \stackrel{f_{s}}{\longrightarrow} X_{s}=X
$$

of nonzero nonisomorphisms between indecomposable modules in $\bmod A$, and the cycle is said to be finite if the homomorphisms $f_{1}, \ldots, f_{s}$ do not belong to the infinite Jacobson radical of $\bmod A$. Then an algebra $A$ is said to be cycle-finite if all cycles in $\bmod A$ are finite AS4. It was shown in [Sk6] that every cycle-finite algebra is of polynomial growth. The class of cycle-finite algebras is wide and contains the algebras of finite type, the tame tilted algebras [Ke, the tame double tilted algebras [RS1, the tame generalized double tilted algebras [RS2], the tame quasi-tilted algebras [LS, Sk10], the tame generalized multicoil algebras [MS, and the strongly simply connected algebras of polynomial growth Sk8]. It has also been proved in AS1, AS2, AS3 that the class of algebras $A$ for which the derived category $D^{b}(\bmod A)$ of bounded complexes over $\bmod A$ is cycle-finite coincides with the class of algebras which are tilting-cotilting equivalent to the hereditary algebras of Dynkin type, hereditary algebras of Euclidean type, or tubular algebras (in the sense of [Ri]), and consequently these algebras $A$ are also cycle-finite. We also mention that by a result due to Peng and Xiao $[\mathrm{PX}]$ and the second named author [Sk4, the Auslander-Reiten quiver of any algebra $A$ has at most finitely many $D \mathrm{Tr}-$ orbits containing directing modules (not lying on a cycle in $\bmod A$ ). Moreover, Ringel has shown that the support algebra of a directing module is a tilted algebra Ri. The support algebras of directing modules over tame algebras have been investigated in [Bo1, Dre] (finite type case) and in [Pe1], Pe2] (representationinfinite case).

The main objective of this paper is to prove that every algebra $A$ which admits a Galois covering $R \rightarrow R / G=A$ with $R$ a cycle-finite locally bounded category and a torsion-free admissible group $G$ of automorphisms of $R$ is tame and to describe the indecomposable finite dimensional $A$-modules. Moreover, we show that, for such Galois coverings, the algebra $A$ is of polynomial growth if and only if the number of $G$-orbits of isoclasses of indecomposable locally finite dimensional $R$-modules with nontrivial stabilizers is finite. As a consequence of the main theorem we also obtain 
that Brenner's conjecture on the number of terms in the middle of almost split sequences over tame algebras is true for the (tame) algebras with cycle-finite Galois coverings.

The main results of the paper extend results proved in Bo2 for algebras of finite type, in [DS3, [ES], $\mathrm{PO}$, [PoS] for biserial algebras, and in [Sk9] for algebras with strongly simply connected (in the sense of [Sk3]) Galois coverings.

The paper is organized as follows. In Section 1 we recall basic facts on Galois coverings of algebras essential for further considerations. Section 2 contains results on cycle-finite algebras applied in the proofs of our main results. Section 3 is devoted to the proof of the main result and its immediate consequences. In Section 4 we establish a criterion for polynomial growth (respectively, domestic type) of algebras with cycle-finite Galois coverings. In the final section, Section 5 , we exhibit examples illustrating our main results.

For basic background on the representation theory of algebras, we refer to the books [ASS, [Ri], [SS1, [SS2].

\section{Galois COverings of Algebras}

Following BoG, by a locally bounded category we mean a $K$-category $R$ which is isomorphic to a factor category $K Q / I$, where $Q$ is a locally finite quiver and $I$ is an admissible ideal of the path category $K Q$ of $Q$. The quiver $Q$ is said to be the quiver of $R$ and will be denoted by $Q_{R}$. A locally bounded category $R$ is said to be connected if its quiver $Q_{R}$ is connected, and triangular if the quiver $Q_{R}$ has no oriented cycles. An algebra $A$ will be considered as a finite category, that is, a locally bounded category given by a finite quiver. A locally bounded category $R$ is said to be interval-finite if, for any two vertices $x$ and $y$ of $Q_{R}$, the number of pairwise different vertices of $Q_{R}$ lying on paths from $x$ to $y$ in $Q_{R}$ is finite. We note that this is a much weaker property than the interval-finiteness defined in $B r G$, (2.6)], which does not force $R$ to be triangular. A full subcategory $C$ of a locally bounded category $R$ is said to be convex if any path in $Q_{R}$ with source and target in $Q_{C}$ lies entirely in $Q_{C}$. Observe that the convex hull of a finite subcategory of an interval-finite category $R$ is a finite convex subcategory of $R$.

Throughout this section we denote by $R$ a fixed locally bounded category (over $K)$. By an $R$-module we mean a contravariant functor $M$ from $R$ to the category MOD $K$ of all vector spaces over $K[\mathrm{BoG}$. An $R$-module $M$ is called finite dimensional (respectively, locally finite dimensional) if $\operatorname{dim} M=\sum_{x \in R} \operatorname{dim}_{K} M(x)<\infty$ (respectively, $\operatorname{dim}_{K} M(x)<\infty$ for any object $x$ of $R$ ). We denote by MOD $R$ the category of all $R$-modules, by $\operatorname{Mod} R(\operatorname{respectively,~} \bmod R)$ the category of all locally finite dimensional (respectively, all finite dimensional) $R$-modules, and by Ind $R$, (respectively, ind $R$ ) the full subcategory of $\operatorname{Mod} R$ (respectively, $\bmod R$ ) formed by all indecomposable modules. The support $\operatorname{supp} M$ of an $R$-module $M$ is the full subcategory of $R$ given by all objects $x$ such that $M(x) \neq 0$.

Let $G$ be a group of $K$-linear automorphisms of $R$ acting freely on the objects of $R$. Then following Ga we may consider the orbit category $R / G$ defined as follows. The objects of $R / G$ are the $G$-orbits of the objects of $R$, and, for any two objects $a$ and $b$ of $R / G$, the morphism $K$-space $(R / G)(a, b)$ is defined as

$$
(R / G)(a, b)=\left\{\left(f_{y, x}\right) \in \prod_{(x, y) \in a \times b} R(x, y) \mid g f_{y, x}=f_{g y, g x} \underset{g \in G, x \in a, y \in b}{\forall}\right\}
$$


and the composition ef of morphisms $f: a \rightarrow b$ and $e: b \rightarrow c$ in $R / G$ is defined by $(e f)_{z x}=\sum_{y \in b} e_{z y} f_{y x}$. Then we have a canonical Galois covering functor

$$
F: R \longrightarrow R / G
$$

which assigns to any object $x$ of $R$ its $G$-orbit $G x$ and maps a morphism $f \in R(x, y)$ onto the family $F(f) \in(R / G)(G x, G y)$ such that $F(f)_{h y, g x}=g f$ or 0 according as $h=g$ or $h \neq g$. Moreover, $F$ induces the $K$-linear isomorphisms

$$
\bigoplus_{F(y)=a} R(x, y) \stackrel{\sim}{\rightarrow}(R / G)(F(x), a), \quad \bigoplus_{F(y)=a} R(y, x) \stackrel{\sim}{\rightarrow}(R / G)(a, F(x)),
$$

for all objects $x$ of $R$ and $a$ of $R / G$. For a full subcategory $D$ of $R$ we denote by $g D$ the full subcategory of $R$ formed by the objects $g x, x \in D$, and its stabilizer $G_{D}=\{g \in G \mid g D=D\}$. Then we may consider the locally bounded category $D / G_{D}$. The group $G$ acts on MOD $R$ by the translations ${ }^{g}(-)$ which assign to each $R$-module $M$ the $R$-module ${ }^{g} M=M \circ g^{-1}$. For each $R$-module $M$, we denote by $G_{M}$ the stabilizer $\left\{g \in G \mid{ }^{g} M \cong M\right\}$ of $M$. Following [DS3, a module $Y$ in Ind $R$ is said to be weakly $G$-periodic if $\operatorname{supp} Y$ is infinite and $(\operatorname{supp} Y) / G_{Y}$ is a finite category. Observe that in such a case, $G_{Y}$ is infinite. We mention that in the later papers of Dowbor [Do1, [Do2, [Do3, Do4, the weakly $G$-periodic modules are called infinite $G$-atoms.

Now assume that $G$ is a group of $K$-linear automorphisms of $R$ acting freely on the isoclasses of modules in ind $R$. Clearly, then $G$ acts freely on the objects of $R$, since $G$ acts freely on the isoclasses of indecomposable projective $R$-modules $R(-, x), x \in R$. Consider the associated Galois covering functor $F: R \rightarrow R / G$. We denote by $F_{\bullet}:$ MOD $R / G \rightarrow \operatorname{MOD} R$ the pull-up functor, which assigns to an $R / G$-module $M$ the $R$-module $M \circ F$, and by $F_{\lambda}:$ MOD $R \rightarrow \operatorname{MOD} R / G$ the push-down functor, left adjoint to $F_{\bullet}$ (see [BoG, (3.2)]). Since $G$ acts freely on the isoclasses in ind $R, F_{\lambda}$ induces an injection from the set (ind $\left.R / \cong\right) / G$ of $G$-orbits of isoclasses in ind $R$ into the set (ind $R / G$ )/ $\cong$ of isoclasses in ind $R / G$ [Ga, (3.5)]. We denote by $\bmod _{1} R / G$ the full subcategory of $\bmod R / G$ consisting of all modules isomorphic to $F_{\lambda}(M)$ for some module $M$ in $\bmod R$, and by $\bmod _{2} R / G$ the full subcategory of $\bmod R / G$ formed by all modules without nonzero direct summands from $\bmod _{1} R / G$. It was shown in [DS3, (2.2) and (2.3)] that a module $X$ from $\bmod R / G$ belongs to $\bmod _{1} R / G$ (respectively, $\left.\bmod _{2} R / G\right)$ if and only if $F_{\bullet}(X)$ is a direct sum of finite dimensional $R$-modules (respectively, weakly $G$-periodic $R$-modules). We denote by $\operatorname{ind}_{1} R / G$ (respectively, $\operatorname{ind}_{2} R / G$ ) the full subcategory of $\bmod _{1} R / G$ (respectively, $\bmod _{2} R / G$ ) formed by the indecomposable modules. Following [DS3 the modules from ind ${ }_{1} R / G$ (respectively, $\operatorname{ind}_{2} R / G$ ) are called indecomposable modules of the first kind (respectively, indecomposable modules of the second kind). The category $R$ is said to be $G$-exhaustive if $\bmod R / G=\bmod _{1} R / G$ [DS3]. From [DS3, (2.5)] we know that $R$ is $G$-exhaustive provided $R$ is locally support-finite, that is, for each object $x$ of $R$, the full subcategory of $R$ consisting of the objects of all supports $\operatorname{supp} M$, where $M$ is a module in ind $R$ with $M(x) \neq 0$, is a finite category. An important class of locally support-finite categories is formed by the locally representation-finite locally bounded categories playing a fundamental role in the representation theory of representation-finite algebras (see BGRS, Bo2, BoG], Ga]). We also mention that if $R$ is $G$-exhaustive, then the Auslander-Reiten quiver $\Gamma_{R / G}$ of $R / G$ is the orbit translation quiver $\Gamma_{R} / G$ of the Auslander-Reiten 
quiver $\Gamma_{R}$ under the induced action of $G$ on $\Gamma_{R}$, because the push-down functor $F_{\lambda}: \bmod R \rightarrow \bmod R / G$ preserves the almost split (Auslander-Reiten) sequences Ga, (3.6)]. The problem whether the $G$-exhaustivity of $R$ forces $R$ to be a locally support-finite category still remains open, although it was confirmed in some important cases (see [AS5], [Sk9]). We refer also to [Do1, [Do2, [Do3, Do4, DS3] for some results on indecomposable $R / G$-modules of the second kind.

A crucial role in the proof of our main result will be played by a reduction theorem from DS3, allowing us to describe the indecomposable modules of the second kind for a class of Galois coverings.

Assume that $R$ is not $G$-exhaustive. Following [DS3, (3.1)], a family $\mathcal{S}$ of full subcategories of $R$ is called separating (with respect to $G$ ) if $\mathcal{S}$ satisfies the following conditions:

(i) for each $L \in \mathcal{S}$ and $g \in G, g L \in \mathcal{S}$;

(ii) for each $L \in \mathcal{S}$ and each $G$-orbit $\mathcal{O}$ of $R, \mathcal{O} \cap L$ is contained in finitely many $G_{L}$-orbits;

(iii) for any two different $L, L^{\prime} \in \mathcal{S}, L \cap L^{\prime}$ is locally support-finite;

(iv) for each weakly $G$-periodic $R$-module $Y$, there exists an $L \in \mathcal{S}$ such that $\operatorname{supp} Y \subseteq L$.

For an additive category $C$ and a full subcategory $C_{0}$ of $C$, we denote by $C /\left[C_{0}\right]$ the factor category of $C$ by the ideal $\left[C_{0}\right]$ of all morphisms in $C$ factorized through a direct sum of some objects of $C_{0}$.

For a convex subcategory $L$ of a locally bounded category $R$ we have a canonical embedding $E^{L}:$ MOD $L \rightarrow$ MOD $R$ such that, for a module $M$ in $\operatorname{MOD} L, E^{L}(N)$ is an $R$-module such that $E^{L}(N)(x)=N(x)$ for any object $x$ of $L, E^{L}(N)(f)=N(f)$ for any morphism $f$ in $L$, and $E^{L}(N)(y)=0$ for any object $y$ of $R$ which is not in $L$. Moreover, for any group $G$ of $K$-linear automorphisms of $R$ acting freely on the objects of $R$, we have a commutative diagram of functors

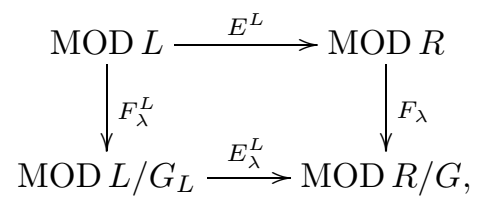

where $F_{\lambda}^{L}$ is the push-down functor associated to the Galois covering $F^{L}: L \rightarrow$ $L / G_{L}, F_{\lambda}$ is the push-down functor associated to the Galois covering $F: R \rightarrow R / G$, and $E_{\lambda}^{L}$ assigns to a module $X$ in $\operatorname{MOD} L / G_{L}$ the module $E_{\lambda}^{L}(X)$ in $\operatorname{MOD} R / G$ such that $F_{\bullet} E_{\lambda}^{L}(X)=\bigoplus_{g \in U_{L}}{ }^{g} F_{\bullet}^{L}(X)$, where $F_{\bullet}:$ MOD $R / G \rightarrow \operatorname{MOD} R$ and $F_{\bullet}^{L}:$ MOD $L / G_{L} \rightarrow$ MOD $L$ are the pull-up functors associated to $F$ and $F^{L}$, and $U_{L}$ is a fixed set of representatives of the cosets of $G$ modulo $G_{L}$ (see [DS3, (2.4), $(3.2)])$.

The following theorem is a special (convex) version of [DS3, Theorem 3.1].

Theorem 1.1. Let $R$ be a locally bounded $K$-category and $G$ a group of $K$-linear automorphisms of $R$ acting freely on the isoclasses in ind $R$. Let $\mathcal{S}$ be a separating family of convex subcategories of $R$ with respect to $G$ and let $\mathcal{S}_{0}$ be a fixed set of representatives of $G$-orbits in $\mathcal{S}$. Then the functors $E_{\lambda}^{L}: \bmod L / G_{L} \rightarrow \bmod R / G$, $L \in \mathcal{S}_{0}$, induce a natural $K$-linear equivalence of categories

$$
E: \coprod_{L \in \mathcal{S}_{0}}\left(\bmod L / G_{L}\right) /\left[\bmod _{1} L / G_{L}\right] \longrightarrow(\bmod R / G) /\left[\bmod _{1} R / G\right]
$$


In particular, the Auslander-Reiten quiver $\Gamma_{R / G}$ of $R / G$ is the disjoint union of the translation quivers

$$
\Gamma_{R / G}=\left(\Gamma_{R} / G\right) \sqcup\left(\coprod_{L \in \mathcal{S}_{0}}\left(\Gamma_{L / G_{L}}\right)_{2}\right),
$$

where $\left(\Gamma_{L / G_{L}}\right)_{2}$ is the union of all connected components of $\Gamma_{L / G_{L}}$ formed by the indecomposable $L / G_{L}$-modules of the second kind.

In this paper we are interested in coverings of algebras by locally bounded categories of tame representation type. Recall that, following Drozd Dro, a finite category $R$ is called tame if, for any dimension $d$, there exists a finite number of $K[T]$ - $R$-bimodules $M_{i}, 1 \leq i \leq n_{d}$, which are finitely generated and free as left $K[T]$-modules, and all but a finite number of isoclasses of indecomposable $R$ modules of dimension $d$ are of the form $K[T] /(T-\lambda) \otimes_{K[T]} M_{i}$ for some $\lambda \in K$ and some $i \in\left\{1, \ldots, n_{d}\right\}$. Let $\mu_{R}(d)$ be the least number of $K[T]-R$-bimodules satisfying the above condition for $d$. Then $R$ is said to be of polynomial growth (respectively, domestic) if there is a positive integer $m$ such that $\mu_{R}(d) \leq d^{m}$ (respectively, $\mu_{R}(d) \leq m$ ) for any $d \geq 1$ (see Sk2, CB2]). An arbitrary locally bounded category $R$ is said to be tame (respectively, of polynomial growth, domestic) if so is every finite full subcategory of $R$. We refer to DS2 for results characterizing tame locally bounded categories. It has been proved in DS1 that if $R$ is a locally bounded category, $G$ a group of automorphisms of $R$ acting freely on the objects of $R$ and $R / G$ is tame, then $R$ is tame. The inverse implication is still an open problem.

The following solution of the stabilizer conjecture by Dowbor in [Do4 is important for applications of covering techniques to tame algebras.

Theorem 1.2. Let $R$ be a tame locally bounded $K$-category and $G$ a torsion-free automorphism group of $R$ acting freely on the objects of $R$. Then the stabilizer $G_{Y}$ of any weakly $G$-periodic $R$-module $Y$ is an infinite cyclic group.

The following consequence of (see [DS3. (2.4), (3.6)]) and [Ga, pp. 94-95] shows the importance of the above theorem for Galois coverings of algebras by tame locally bounded categories.

We note that the restriction imposed on the group to be torsion-free is natural because of the results of $\mathrm{Ga}$, GP].

Proposition 1.3. Let $R$ be a locally bounded $K$-category, $G$ a group of $K$-linear automorphisms of $R$ acting freely on the objects of $R$, and $Y$ a weakly $G$-periodic $R$-module whose stabilizer $G_{Y}$ is an infinite cyclic group. Then $F_{\lambda}(Y)$ carries a canonical structure of a $K G_{Y}-R / G$-bimodule which is a free module of finite rank as a left module over the group algebra $K G_{Y}$ of $G_{Y}$. In particular, we have a canonical functor

$$
\Phi^{Y}=-\otimes_{K G_{Y}} F_{\lambda}(Y): \bmod K G_{Y} \longrightarrow \bmod R / G
$$

whose image is contained in $\bmod _{2} R / G$.

We also note that, if $G_{L}$ is infinite cyclic, then $K G_{L}$ is isomorphic to the algebra $K\left[T, T^{-1}\right]$ of Laurent polynomials.

Let $R$ be a locally bounded $K$-category and $G$ a group of $K$-linear automorphisms of $R$ acting freely on the objects of $R$. A line in $R$ is a convex subcategory $L$ of $R$ 
which is isomorphic to the path category $K Q$ of a linear quiver $Q$ of type $\mathbb{A}_{n}, \mathbb{A}_{\infty}$ or $\infty A_{\infty}$. A line $L$ in $R$ is said to be $G$-periodic if its stabilizer $G_{L}$ is nontrivial. Clearly, in this case, the quiver $Q_{L}$ of $L$ is of type

$$
{ }_{\infty} \mathbb{A}_{\infty}: \quad \ldots \longrightarrow \bullet-\bullet-\bullet-\bullet
$$

and has a $G_{L}$-periodic orientation. With each $G$-periodic line $L$ of $R$ we may associate a canonical weakly $G$-periodic $R$-module $M_{L}$ by setting $M_{L}(x)=K$ for any vertex $x$ of $Q_{L}, M_{L}(y)=0$ for all vertices $y$ of $Q_{R} \backslash Q_{L}$, and $M_{L}(\gamma)=\operatorname{id}_{K}$ for each arrow $\gamma$ of $Q_{L}$. Since $G_{M_{L}}=G_{L}=\mathbb{Z}$, we then obtain a canonical functor

$$
\Phi^{L}=-\otimes_{K\left[T, T^{-1}\right]} F_{\lambda}\left(M_{L}\right): \bmod K\left[T, T^{-1}\right] \longrightarrow \bmod R / G,
$$

where $\bmod K\left[T, T^{-1}\right]$ denotes the category of finite dimensional modules over $K\left[T, T^{-1}\right]$.

\section{CyCle-Finite Algebras}

In this section we describe properties of cycle-finite algebras applied in the proof of our main theorem and its consequences.

By a tame concealed algebra we mean a tilted algebra $C=\operatorname{End}_{H}(T)$, where $H$ is the path algebra $K Q$ of a quiver $Q$ of Euclidean type $\widetilde{\mathbb{A}}_{m}(m \geq 1), \widetilde{\mathbb{D}}_{n}(n \geq 4)$, or $\widetilde{\mathbb{E}}_{p}(6 \leq p \leq 8)$, and $T$ is a (multiplicity-free) preprojective tilting $H$-module. The tame concealed algebras have been completely classified by quivers and relations in [Bo3] and [HV]. Recall also that the Auslander-Reiten quiver $\Gamma_{C}$ of a tame concealed algebra $C$ is of the form

$$
\Gamma_{C}=\mathcal{P}^{C} \vee \mathcal{T}^{C} \vee \mathcal{Q}^{C},
$$

where $\mathcal{P}^{C}$ is a preprojective component containing all indecomposable projective $C$ modules, $\mathcal{Q}^{C}$ is a preinjective component containing all indecomposable injective $C$ modules, and $\mathcal{T}^{C}$ is a $\mathbb{P}_{1}(K)$-family $\mathcal{T}_{\lambda}^{C}, \lambda \in \mathbb{P}_{1}(K)$, of pairwise orthogonal standard stable tubes, all but a finite number of them of rank one (see [Ri, Chapter 4] and [SS1] for further properties of the module category of a tame concealed algebra).

By a tubular algebra we mean a tubular extension (equivalently, tubular coextension) of a tame concealed algebra of tubular type $(2,2,2,2),(3,3,3),(2,4,4)$, or $(2,3,6)$, as defined in $\mathrm{Ri}$. Recall that a tubular algebra $B$ admits two different tame concealed convex subcategories $C_{0}$ and $C_{\infty}$ such that the Auslander-Reiten quiver $\Gamma_{B}$ of $B$ is of the form

$$
\Gamma_{B}=\mathcal{P}_{0}^{B} \vee \mathcal{T}_{0}^{B} \vee\left(\bigvee_{q \in \mathbb{Q}^{+}} \mathcal{T}_{q}^{B}\right) \vee \mathcal{T}_{\infty}^{B} \vee Q_{\infty}^{B},
$$

where $\mathcal{P}_{0}^{B}$ is the preprojective component $\mathcal{P}^{C_{0}}$ of $\Gamma_{C_{0}}, \mathcal{T}_{0}^{B}$ is a $\mathbb{P}_{1}(K)$-family of pairwise orthogonal standard ray tubes, obtained from the stable tubes of $\mathcal{T}^{C_{0}}$ by ray insertions, $Q_{\infty}^{B}$ is the preinjective component $Q^{C_{\infty}}$ of $\Gamma_{C_{\infty}}, \mathcal{T}_{\infty}^{B}$ is a $\mathbb{P}_{1}(K)$-family of pairwise orthogonal standard coray tubes, obtained from the stable tubes of $\mathcal{T}^{C_{\infty}}$ by coray insertions, and, for each $q \in \mathbb{Q}^{+}$(the set of positive rational numbers), $\mathcal{T}_{q}^{B}$ is a $\mathbb{P}_{1}(K)$-family of pairwise orthogonal standard stable tubes (we refer to $\mathrm{Ri}$, Chapter 5] for more properties of module categories of tubular algebras).

Recall also that a module $M$ over an algebra $A$ is called sincere if $\operatorname{supp} M=A$.

The following characterization of tame concealed and tubular algebras was established in [Sk6, Theorem 4.1]. 
Theorem 2.1. Let $A$ be an algebra. The following conditions are equivalent:

(i) $A$ is cycle-finite and admits a sincere indecomposable module lying in a stable tube of $\Gamma_{A}$.

(ii) $A$ is either tame concealed or tubular.

Moreover, we have the following consequence of the above theorem and a result of Crawley-Boevey [CB1, Corollary E], proved in [Sk6, Theorem 4.3].

Theorem 2.2. Let $A$ be a cycle-finite algebra. Then $A$ is of polynomial growth.

An algebra $A$ is said to be minimal representation-infinite if $A$ is representationinfinite but every proper convex subcategory of $A$ is representation-finite. Then we have the following characterization of minimal representation-infinite cycle-finite algebras established in [Sk6, Corollary 4.4].

Theorem 2.3. For an algebra $A$ the following conditions are equivalent:

(i) A is minimal representation-infinite and cycle-finite.

(ii) A is tame concealed.

We also mention the following characterization of domestic cycle-finite algebras, proved in [Sk6, Theorem 5.1].

Theorem 2.4. Let $A$ be a cycle-finite algebra. The following conditions are equivalent:

(i) A is domestic.

(ii) A does not contain a tubular algebra as a convex subcategory.

(iii) All but finitely many components of $\Gamma_{A}$ are stable tubes of rank one.

Let $A$ be a cycle-finite algebra. The support supp $M$ of a module $M$ in ind $A$ is not necessarily a convex subcategory of $A$. We denote by $c(M)$ the number of pairwise different tame concealed convex subcategories of the convex hull of supp $M$ in $A$.

The following fact has recently been established in our joint work with Malicki [MPS, Theorem B].

Theorem 2.5. Let $A$ be a cycle-finite algebra and $M$ be an indecomposable finite dimensional A-module. Then $c(M) \leq 3$.

We note that there are many cycle-finite algebras $A$ having indecomposable finite dimensional modules $M$ with $c(M)=3$. For example, the iterated tubular algebras studied in $\mathrm{PTO}$ have this property.

We also mention that there are (discrete) indecomposable finite dimensional modules over cycle-finite algebras with a very complicated structure, for example the modules lying in generalized multicoils of tame generalized multicoil algebras (see $[\mathrm{MS}]$ ).

We end this section with a result on the number of terms in the middle of almost split sequences over cycle-finite algebras.

For an algebra $A$ and a nonprojective indecomposable module $X$ in $\bmod A$, consider an almost split sequence

$$
0 \longrightarrow \tau_{A} X \longrightarrow \bigoplus_{i=1}^{s(X)} Y_{i} \longrightarrow X \longrightarrow 0
$$


where all $Y_{i}$ are indecomposable $A$-modules. It has been proved by Bautista and Brenner [BB] (see also [Li]) that if $A$ is representation-finite, then $s(X) \leq 4$, and if $s(X)=4$, then one of the modules $Y_{i}$ is projective-injective. It has been conjectured by Brenner that, for a tame algebra $A, s(X) \leq 5$ for every nonprojective indecomposable finite dimensional $A$-module $X$. This has been confirmed for some classes of tame algebras but for arbitrary tame algebras it is still an open and interesting problem.

For cycle-finite algebras this was shown in [PTa, Theorem 3] as follows.

Theorem 2.6. Let $A$ be a cycle-finite algebra and

$$
0 \longrightarrow \tau_{A} X \longrightarrow \bigoplus_{i=1}^{s(X)} Y_{i} \longrightarrow X \longrightarrow 0
$$

be an almost split sequence in $\bmod A$, where the modules $Y_{i}$ are indecomposable. Then $s(X) \leq 5$. Moreover, if $s(X)=5$, then one of the modules $Y_{i}$ is projectiveinjective.

We recall that an almost split sequence in $\bmod A$ whose middle term contains an indecomposable projective-injective module $P$ is isomorphic to the almost split sequence of the form

$$
0 \longrightarrow \operatorname{rad} P \longrightarrow(\operatorname{rad} P / \operatorname{soc} P) \oplus P \longrightarrow P / \operatorname{soc} P \longrightarrow 0
$$

(see [ASS, Proposition 3.11]).

\section{The Main Result AND CONSEQUences}

A locally bounded $K$-category $R$ is said to be cycle-finite if $R$ is interval-finite and every finite convex subcategory of $R$ is cycle-finite. A group $G$ of $K$-linear automorphisms of a locally bounded category $R$ is said to be admissible if its action on the objects of $R$ is free and has finitely many orbits. In such a case, $R / G$ is a finite category (algebra). For a locally bounded category $R$ and an admissible group $G$ of automorphisms of $R$, we denote by $\mathcal{A}(R, G)$ a fixed set of representatives of isoclasses of weakly $G$-periodic $R$-modules closed under the induced action of $G$, and by $\mathcal{A}_{0}(R, G)$ a fixed set of representatives of $G$-orbits in $\mathcal{A}(R, G)$. Moreover, we identify the group algebra $K \mathbb{Z}$ of the infinite cyclic group $\mathbb{Z}$ with the algebra $K\left[T, T^{-1}\right]$ of Laurent polynomials.

The following theorem is the main result of the paper.

Theorem 3.1. Let $R$ be a connected cycle-finite locally bounded $K$-category over an algebraically closed field $K, G$ a torsion-free admissible group of $K$-linear automorphisms of $R, A=R / G, \mathcal{A}=\mathcal{A}(R, G)$, and $\mathcal{A}_{0}=\mathcal{A}_{0}(R, G)$. Then the functors $\Phi^{Y}=-\otimes_{K\left[T, T^{-1}\right]} F_{\lambda}(Y): \bmod K\left[T, T^{-1}\right] \rightarrow \bmod A, Y \in \mathcal{A}_{0}$, induce a K-linear equivalence of categories

$$
\Phi: \coprod_{\mathcal{A}_{0}} \bmod K\left[T, T^{-1}\right] \stackrel{\sim}{\longrightarrow} \bmod A /\left[\bmod _{1} A\right] .
$$

Moreover, the following statements hold:

(i) $A$ is tame.

(ii) Every indecomposable finite dimensional A-module $X$ is isomorphic either to $F_{\lambda}(M)$ for some indecomposable finite dimensional $R$-module $M$ 
or to $\Phi^{Y}(V)$ for some $Y \in \mathcal{A}_{0}$ and some indecomposable finite dimensional $K\left[T, T^{-1}\right]$-module $V$.

(iii) The Auslander-Reiten quiver $\Gamma_{A}$ of $A$ has the disjoint union decomposition

$$
\Gamma_{A}=\left(\Gamma_{R} / G\right) \sqcup\left(\coprod_{\mathcal{A}_{0}} \Gamma_{K\left[T, T^{-1}\right]}\right),
$$

where $\Gamma_{K\left[T, T^{-1}\right]}$ is the Auslander-Reiten quiver of the category of finite dimensional $K\left[T, T^{-1}\right]$-modules.

Proof. Since $G$ is torsion-free and acts freely on the objects of $R$, it acts freely on the isoclasses of modules in ind $R$. Then the push-down functor $F_{\lambda}: \bmod R \rightarrow \bmod A$ preserves the Auslander-Reiten sequences and induces an injection from the set of $G$-orbits of isoclasses in ind $R$ into the set of isoclasses in ind $A$ [Ga, Section 3]. We will show that the functors $\Phi^{Y}, Y \in \mathcal{A}_{0}$, induce a $K$-linear equivalence of categories

$$
\Phi: \coprod_{\mathcal{A}_{0}} \bmod K\left[T, T^{-1}\right] \longrightarrow \bmod A /\left[\bmod _{1} A\right]
$$

applying Theorems [1.1, 2.3 and 2.5, and results on representation-finite algebras. Observe that this will imply that every indecomposable module $X$ in $\operatorname{ind}_{2} A$ is isomorphic to a module $\Phi^{Y}(V)$ for some $Y \in \mathcal{A}_{0}$ and some indecomposable finite dimensional $K\left[T, T^{-1}\right]$-module $V$, and consequently (ii) will be proved.

In the first step of our proof we describe the structure of modules in Ind $R$ with nontrivial stabilizer, applying the theory of fundamental sequences developed in DS3, Section 4]. We need a family of finite convex subcategories of $R$.

Following DS1, for a full subcategory $C$ of $R$, we denote by $\widehat{C}$ the full subcategory of $R$ formed by all objects $x$ such that $R(x, y) \neq 0$ or $R(y, x) \neq 0$ for some object $y$ of $C$. Observe that, if $C$ is finite, then $\widehat{C}$ is also finite, because $R$ is locally bounded. For an $R$-module $Z$ and a full subcategory $C$ of $R$, we denote by $\left.Z\right|_{C}$ the restriction of $Z$ to $C$.

Fix a family $C_{n}, n \in \mathbb{N}$, of finite convex subcategories of $R$ such that:

(1) For each $n \in \mathbb{N}, C_{n+1}$ is the convex hull of $\widehat{C}_{n}$ in $R$.

(2) $R=\bigcup_{n \in \mathbb{N}} C_{n}$.

Since $R$ is connected, locally bounded and interval-finite, such a family exists. We shall identify a $C_{n}$-module $M$ with an $R$-module, by setting $M(x)=0$ for all objects $x$ of $R$ which are not in $C_{n}$.

Let $Y$ be a module in Ind $R$ with $G_{Y}$ nontrivial. We will show that $Y$ is a weakly $G$-periodic $R$-module and describe its support $\operatorname{supp} Y$. Since $R$ is cycle-finite, hence tame, Theorem 1.2 will then imply that $G_{Y}$ is an infinite cyclic group.

Let $m \in \mathbb{N}$ be the least number such that $\left.Y\right|_{C_{m}} \neq 0$. We define a family of modules $Y_{n} \in$ ind $C_{n}, n \in \mathbb{N}$, as follows. We set $Y_{n}=0$ for $n<m$, and let $Y_{m}$ be an arbitrary indecomposable direct summand of $\left.Y\right|_{C_{m}}$. Then there exist $Y_{m+1} \in$ ind $C_{m+1}$ and a splittable monomorphism $u_{m}:\left.Y_{m} \rightarrow Y_{m+1}\right|_{C_{m}}$ such that $Y_{m+1}$ is a direct summand of $\left.Y\right|_{C_{m+1}}$. Repeating this procedure we can find, for all $n \geq m, Y_{n} \in \operatorname{ind} C_{n}$ and splittable monomorphisms $u_{n}:\left.Y_{n} \rightarrow Y_{n+1}\right|_{C_{n}}$ such that $Y_{n}$ is a direct summand of $\left.Y\right|_{C_{n}}$. We obtain a sequence $\left(Y_{n}, u_{n}\right)_{n \in \mathbb{N}}$, called in DS3, (4.1)], a fundamental $R$-sequence produced by $Y$. Since $C_{n}, n \in \mathbb{N}$, are finite convex subcategories of $R$ and $Y$ is a locally finite dimensional $R$-module, it is in fact a sequence of finite dimensional indecomposable $R$-modules. Now invoking the facts 
that $Y \in$ Ind $R$ and $Y \cong{ }^{g} Y$, we have the following consequences of [DS3. (4.3), $(4.4),(4.5)]$ :

(a) $Y=\lim Y_{n}$.

(b) For each $n \in \mathbb{N}$, there exists $p \geq n$ such that $\left.\left.Y_{p}\right|_{C_{n}} \cong Y\right|_{C_{n}}$.

(c) For each $g \in G_{Y}$ and $n \in \mathbb{N}$, there exists $q \geq n$ such that $g C_{n} \subseteq C_{q}$ and ${ }^{g} Y_{n}$ is a direct summand of $\left.Y_{q}\right|_{g C_{n}}$.

For $n \geq m$, we denote by $B_{n}$ the convex hull of supp $Y_{n}$ in $R$. Clearly, $B_{n} \subseteq C_{n}$ for any $n \geq m$. Moreover, since $Y$ is indecomposable, infinite dimensional, locally finite dimensional, and $C_{n+1}$ contains $\widehat{C}_{n}$, for each $n \in \mathbb{N}$, applying [DS1, Lemma 2], we conclude that, for any $n \geq m, B_{n}$ is not contained in $C_{n-1}$.

We now claim that all categories $B_{n}, n \geq m$, are representation-finite. Assume that $B_{p}$, for some $p \geq m$, is representation-infinite. Then, by Theorem $2.3, B_{p}$ contains a tame concealed algebra $D$ as a convex subcategory, because $B_{p} \subseteq C_{p}$ and $C_{p}$ is cycle-finite, as a convex subcategory of $R$. Take an arbitrary $r \in \mathbb{N}$ and consider the tame concealed convex subcategories $D, g D, \ldots, g^{r} D$, for a fixed $g \in$ $G_{Y} \backslash\left\{1_{D}\right\}$. Since the group $G$ is torsion-free and acts freely on the objects of $R$, the categories $D, g D, \ldots, g^{r} D$ are pairwise different. It follows from (c) that, for each $i \in\{0, \ldots, r\}$, there exists $t_{i} \geq p$ such that $g^{i} C_{p} \subseteq C_{t_{i}}$ and $g^{i} Y_{p}$ is a direct summand of $Y_{t_{i}} \mid g_{g^{i} C_{p}}$. Take $q \in \mathbb{N}$ such that $C_{q}$ contains all categories $C_{t_{i}}, i \in\{0, \ldots, r\}$. Then $Y_{t_{i}}$ is a direct summand of $\left.Y_{q}\right|_{C_{t_{i}}}$, and hence ${ }^{g^{i}} Y_{p}$ is a direct summand of $\left.Y_{q}\right|_{g^{i} C_{p}}$, for any $i \in\{0, \ldots, r\}$. Therefore, $Y_{q}$ is an indecomposable finite dimensional $R$ module for which the convex hull $B_{q}$ of $\operatorname{supp} Y_{q}$ contains pairwise different tame concealed convex subcategories $D, g D, \ldots, g^{r} D$. On the other hand, $C_{q}$ is a cyclefinite algebra containing $B_{q}$ as a convex subcategory, and so, applying Theorem 2.5. we conclude that $B_{q}$ contains at most 3 pairwise different tame concealed convex subcategories. Hence, taking $r \geq 3$, we get a contradiction. Indeed this shows that all categories $B_{n}, n \geq m$, are representation-finite.

Our next claim is to show that all categories $B_{n}, n \geq m$, are standard, that is, are isomorphic to their standard forms $\bar{B}_{n}$ (see [BoG, (5.1)] and [BrG, (3.1)]). Assume that $B_{p}$, for some $p \geq m$, is nonstandard. Then, by [BGRS, Theorem 9.6], $K$ is of characteristic 2 and $B_{p}$ contains a convex subcategory $P$ which is a penny-farthing (Riedtmann-contour) $K Q / I$ given by the quiver $Q$ of the form

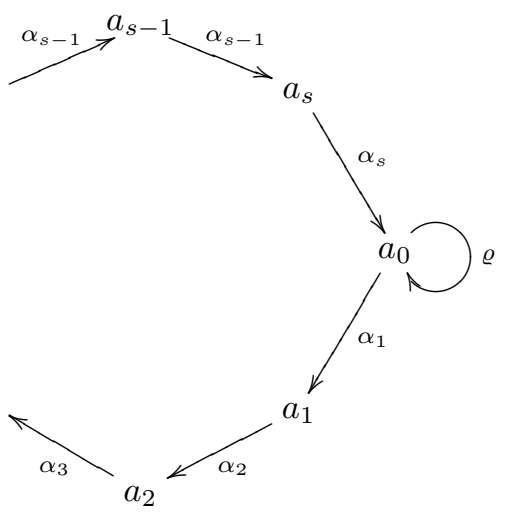

for some $s \geq 2$, and $I$ is an ideal of the path algebra $K Q$ of $Q$ generated by the elements $\alpha_{1} \ldots \alpha_{s}-\varrho^{2}, \alpha_{s} \alpha_{1}-\alpha_{s} \varrho \alpha_{1}$, and $\alpha_{i+1} \ldots \alpha_{n} \varrho \alpha_{1} \ldots \alpha_{f(i)}, 1 \leq i \leq s-1$, 
where $f$ is a nondecreasing function from $\{1, \ldots, s-1\}$ to $\{1, \ldots, s\}$, which is not constant with value 1 . Moreover, we may assume that $p \geq m+14$. Take an arbitrary positive integer $r \geq 3$ and consider the convex subcategories $P, g P, \ldots, g^{r} P$ of $P$, which are clearly pairwise different, since $G$ is torsion-free and acts freely on the objects of $R$. We conclude as above that there exists $q \geq p$ such that the convex hull $B_{q}$ of $\operatorname{supp} Y_{q}$ in $R$ contains all the categories $P, g P, \ldots, g^{r} P$ as convex subcategories. It has been proved by Bongartz in [Bo4, Corollary 3] that every representation-finite algebra $\Lambda$ which admits a faithful indecomposable module is standard. Therefore, the indecomposable $B_{q}$-module $Y_{q}$ is an indecomposable module over the standard algebra $\bar{B}_{q}$ of $B_{q}$ in which (see [BGRS, 2.7] and BrG, (3.1)]) the nonstandard convex subcategories $P, g P, \ldots, g^{r} P$ of $B_{q}$ are replaced by their standard forms $\bar{P}, g \bar{P}, \ldots, g^{r} \bar{P}$, where $\bar{P}=K Q / \bar{I}$ is given by the same pennyfarthing quiver $Q$, and $\bar{I}$ is the ideal in $K Q$ generated by the elements $\alpha_{1} \ldots \alpha_{s}-\varrho^{2}$, $\alpha_{s} \alpha_{1}$, and $\alpha_{i+1} \ldots \alpha_{n} \varrho \alpha_{1} \ldots \alpha_{f(i)}, 1 \leq i \leq s-1$. According to [BrG, Theorem 3.1], $\bar{B}_{q}=K Q_{\bar{B}_{q}} / I_{\bar{B}_{q}}$, where $Q_{\bar{B}_{q}}$ is the quiver of $\bar{B}_{q}$ and the ideal $I_{\bar{B}_{q}}$ of $K Q_{\bar{B}_{q}}$ is generated by all nonstable paths of $Q_{\bar{B}_{q}}$ and the differences $v-w$, where $(v, w)$ ranges over the stable contours of $\bar{B}_{q}$ (see [BrG, (1.5)]). Now the representation-finite standard algebra $\Lambda_{q}=\bar{B}_{q}$ admits a simply connected locally representation-finite universal Galois covering $F^{q}: \widetilde{\Lambda}_{q} \rightarrow \widetilde{\Lambda}_{q} / G_{q}=\Lambda$ with the free fundamental group $G_{q}=\Pi_{1}\left(Q_{\bar{B}_{q}}, I_{\bar{B}_{q}}\right)$, where $\widetilde{\Lambda}_{q}=\widetilde{Q}_{\bar{B}_{q}} / \widetilde{I}_{\bar{B}_{q}}$, as defined in [MP, Corollary 1.5] (see also [Ga, (2.1)]). Since $\widetilde{\Lambda}_{q}$ is locally representation-finite, the associated push-down functor $F_{\lambda}^{q}: \bmod \widetilde{\Lambda}_{q} \rightarrow \bmod \Lambda_{q}$ is dense. In particular, the indecomposable $\Lambda_{q^{-}}$ module $Y_{q}$ is isomorphic to $F_{\lambda}^{q}\left(M_{q}\right)$ for some indecomposable finite dimensional $\widetilde{\Lambda}_{q}$-module $M_{q}$. Moreover, the support supp $M_{q}$ of $M_{q}$ is a finite convex subcategory of $\widetilde{\Lambda}_{q}$ containing at least 14 objects (by our choice of $p$ ) and contains pairwise different convex subcategories $E_{0}, E_{1}, \ldots, E_{r}$ each of them isomorphic to the path category $K \Delta / J$, where $\Delta$ is the quiver

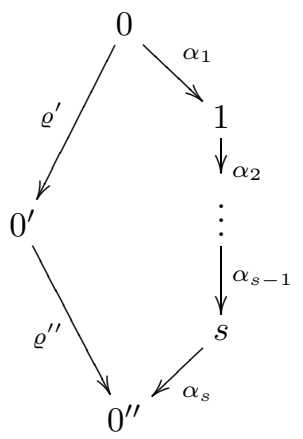

and $J$ is the ideal of $K \Delta$ generated by $\alpha_{1} \ldots \alpha_{s}-\varrho^{\prime} \varrho^{\prime \prime}$. This contradicts the shapes of the quivers on the Bongartz list [Bo1, (2.4)] of 24 families of algebras forming the support algebras of all indecomposable modules over the representation-finite simply connected algebras having quivers with at least 14 vertices (see also Ri, (6.3)]). Therefore, indeed all algebras $B_{n}, n \geq m$, are representation-finite and standard.

We will now describe the convex hull $B=\bigcup_{n \geq m} B_{n}$ of the support $\operatorname{supp} Y$ of $Y=\underline{\lim } Y_{n}$. Take $p=m+14$. For each $n \geq p$, consider the universal 
Galois covering $F^{n}: \widetilde{B}_{n} \rightarrow \widetilde{B}_{n} / G_{n}=B_{n}$, given by the standard form presentation $B_{n}=K Q_{B_{n}} / I_{B_{n}}$ described in [BrG, (3.1)], the fundamental group $G_{n}=\Pi_{1}\left(Q_{B_{n}}, I_{B_{n}}\right)$ of the bound quiver $\left(Q_{B_{n}}, I_{B_{n}}\right)$, and $\widetilde{B}_{n}=K \widetilde{Q}_{B_{n}} / \widetilde{I}_{B_{n}}$ the associated simply connected, locally representation-finite, locally bounded category. Since the push-down functors $F_{\lambda}^{n}: \bmod \widetilde{B}_{n} \rightarrow \bmod B_{n}$, are dense, for each $n \geq p$, we have $Y_{n}=F_{\lambda}^{n}\left(M_{n}\right)$ for an indecomposable finite dimensional $\widetilde{B}_{n}$-module $M_{n}$. Observe also that, for any $n \geq p$, the support $D_{n}=\operatorname{supp} M_{n}$ of $M_{n}$ in $\widetilde{B}_{n}$ has at least $p=m+14 \geq 14$ objects. It follows from the property (c) of the fundamental $R$-sequence $\left(Y_{n}, u_{n}\right)_{n \in \mathbb{N}}$ produced by $Y$ that, for each $g \in G_{Y} \backslash\{1\}$ and $n \geq p$, there exists $r \geq n$ such that $g C_{n} \subseteq C_{r},{ }^{g} Y_{n}$ is a direct summand of $\left.Y_{r}\right|_{g C_{n}}$, and obviously $Y_{n}$ is a direct summand of $\left.Y_{r}\right|_{C_{n}}$. Again using the structure of indecomposable sincere modules over Bongartz's 24 families of algebras from [Bo1, (2.4)] and the fact that $M_{n}$ are sincere indecomposable $D_{n}$-modules with $F_{\lambda}^{n}\left(M_{n}\right)=Y_{n}$, we conclude that all categories $D_{n}, n \geq p$, are finite convex lines of $\widetilde{B}_{n}$, and $M_{n}$ are isomorphic to the canonical linear indecomposable modules $M_{D_{n}}$ associated to the lines $D_{n}$, respectively. Moreover, we obtain $B_{n}=F^{n}\left(D_{n}\right)=F^{n}\left(\operatorname{supp} M_{n}\right)=\operatorname{supp} Y_{n}$, for any $n \geq p$, and hence $B_{n}=\operatorname{supp} Y_{n}$ for any $n \geq m$. This implies that $B=\operatorname{supp} Y$ is a convex subcategory which admits a simply connected (not locally representationfinite) Galois covering $F^{B}: \widetilde{B} \rightarrow \widetilde{B} / H=B$ with a free group $H$ such that $Y \cong F_{\lambda}^{B}\left(M_{L}\right)$ for the canonical linear module $M_{L}$ over a convex line $L$ of type $\infty_{\infty}$ of $\widetilde{B}$ with nontrivial stabilizer $H_{L}=\{h \in H \mid h L=L\}$. As a consequence we obtain that $\operatorname{supp} Y / G_{L}=B / G_{L}$ is a finite category, since $L / H_{L}$ is a finite category. In particular, we conclude that $Y$ is a representative of the unique isoclass of modules $Z$ in Ind $R$ with nontrivial stabilizer $G_{Z}$ and $\operatorname{supp} Z \subseteq B=\operatorname{supp} Y$. Obviously, $Y$ is a weakly $G$-periodic $R$-module.

Summing up, we have proved that the modules $Y$ in Ind $R$ with nontrivial stabilizer $G_{Y}$ are weakly $G$-periodic $R$-modules, $G_{Y}$ are infinite cyclic groups, $B_{Y}=$ supp $Y$ are convex subcategories of $R$ with $G_{B_{Y}}=G_{Y}$, uniquely determined by the isoclass of $Y$, and $Y$ is isomorphic to the push-down $F_{\lambda}^{B_{Y}}\left(M_{L}\right)$ of the canonical linear indecomposable module $M_{L}$, where $F_{\lambda}^{B_{Y}}: \operatorname{Mod} \widetilde{B}_{Y} \rightarrow \operatorname{Mod} B_{Y}$ is the pushdown functor associated to a universal Galois covering $F^{B_{Y}}: \widetilde{B}_{Y} \rightarrow \widetilde{B}_{Y} / H_{Y}=B_{Y}$, and $L$ is an $H_{Y}$-periodic line in $\widetilde{B}_{Y}$. Moreover, every finite convex subcategory of $B_{Y}=\operatorname{supp} Y$ is representation-finite.

Assume now that $R$ is not $G$-exhaustive, equivalently that there exist weakly $G$-periodic $R$-modules. Denote by $\mathcal{S}$ the family of the supports $B_{Y}$ of all weakly $G$-periodic $R$-modules $Y$. We claim that $\mathcal{S}$ is a separating family of convex subcategories of $R$, and hence Theorem 1.1 can be applied. Clearly, if $Y$ is a weakly $G$-periodic $R$-module and $g \in G$, then ${ }^{g} Y$ is a weakly $G$-periodic $R$-module with $B_{g_{Y}}=\operatorname{supp}\left({ }^{g} Y\right)=g B_{Y}=g \operatorname{supp} Y$ and $G_{g_{Y}}=g G_{Y} g^{-1}$, and hence the condition (i) is satisfied. For any $G$-orbit $\mathcal{O}$ of $R$, the intersection $\mathcal{O} \cap B_{Y}$ is contained in finitely many $G_{Y}$-orbits, because $B_{Y} / G_{Y}$ is finite, so (ii) holds. Further, for two different categories $B_{Y}$ and $B_{Y^{\prime}}$ in $\mathcal{S}$, the intersection $B_{Y} \cap B_{Y^{\prime}}$ is a finite, and hence representation-finite, convex subcategory of $B_{Y}$ (and of $B_{Y^{\prime}}$ ), and so (iii) holds. The condition (iv) is trivially satisfied.

Now consider a fixed set $\mathcal{A}=\mathcal{A}(R, G)$ of representatives of isoclasses of weakly $G$-periodic $R$-modules closed under the induced action of $G$, and a fixed set $\mathcal{A}_{0}=$ $\mathcal{A}_{0}(R, G)$ of representatives of $G$-orbits in $\mathcal{A}(R, G)$. Observe that then the supports 
$B_{Y}=\operatorname{supp} Y$ of all modules $Y$ in $\mathcal{A}_{0}$ form a set $\mathcal{S}_{0}$ of representatives of $G$-orbits of the separating family $\mathcal{S}$ of convex subcategories of $R$ defined above. Therefore, applying Theorem 1.1 we have a commutative diagram of functors

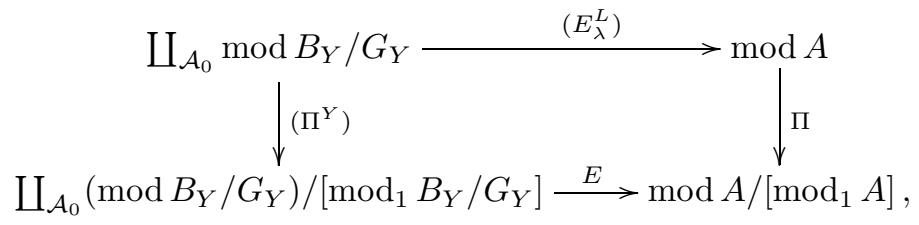

where $E_{\lambda}^{L}: \bmod B_{Y} / G_{Y} \rightarrow \bmod A=\bmod R / G$ are the functors induced by the embeddings $E^{Y}: \operatorname{MOD} B_{Y} \rightarrow \operatorname{MOD} R$ (described in Section 1), $E$ is a $K$-linear equivalence of categories, and $\Pi^{Y}: \bmod B_{Y} / G_{Y} \rightarrow\left(\bmod B_{Y} / G_{Y}\right) /\left[\bmod _{1} B_{Y} / G_{Y}\right]$ and $\Pi: \bmod A \rightarrow(\bmod A) /\left[\bmod _{1} A\right]$ are the canonical quotient functors.

Now take a module $Y$ in $\mathcal{A}_{0}$ and consider the universal Galois covering functor $F^{B_{Y}}: \widetilde{B}_{Y} \rightarrow \widetilde{B}_{Y} / H_{Y}=B_{Y}$ of the support $B_{Y}=\operatorname{supp} Y$ of $Y$, and the associated push-down functor $F_{\lambda}^{B_{Y}}: \operatorname{MOD} \widetilde{B}_{Y} \rightarrow \operatorname{MOD} B_{Y}$. Then $Y \cong F_{\lambda}^{B_{Y}}\left(M_{L}\right)$ for the canonical linear $\widetilde{B}_{Y}$-module $M_{L}$ associated to an $H_{Y}$-periodic line $L$ of $\widetilde{B}_{Y}$. It follows from the above discussion that there is only one $H_{Y}$-orbit of $H_{Y}$-periodic lines in $\widetilde{B}_{Y}$, so we may fix an $H_{Y}$-periodic line $L_{Y}$ in $\widetilde{B}_{Y}$ and the linear $\widetilde{B}_{Y^{-}}$ module $M_{Y}=M_{L_{Y}}$ such that $Y=F_{\lambda}^{B_{Y}}\left(M_{Y}\right)$ and $B_{Y}=F^{B_{Y}}\left(L_{Y}\right)$. Denote by $F^{Y}: B_{Y} \rightarrow B_{Y} / G_{Y}$ the Galois covering functor induced by the action of $G_{Y}$ on $B_{Y}$ and by $F_{\lambda}^{Y}: \operatorname{MOD} B_{Y} \rightarrow \operatorname{MOD} B_{Y} / G_{Y}$ the associated push-down functor. Consider the composed Galois covering functor

$$
\mathbb{F}^{Y}=F^{Y} \circ F^{B_{Y}}: \widetilde{B}_{Y} \rightarrow B_{Y} / G_{Y}=\widetilde{B}_{Y} / \mathbb{G}_{Y}
$$

which is given by an admissible group $\mathbb{G}_{Y}$ of $K$-linear automorphisms of $\widetilde{B}_{Y}$ having $H_{Y}$ as a normal subgroup with $\mathbb{G}_{Y} / H_{Y}=G_{Y}$ (see [Ga]). Moreover, $\widetilde{B}_{Y}$ is a simply connected locally bounded category whose every finite convex subcategory is representation-finite. Then, applying [DS3, Proposition 5.1], we obtain that every weakly $\mathbb{G}_{Y}$-periodic $\widetilde{B}_{Y}$-module $Z$ is linear. Clearly, the push-down $F_{\lambda}^{B_{Y}}(Z)$ of such a weakly $\mathbb{G}_{Y}$-periodic $\widetilde{B}_{Y}$-module $Z$ is a weakly $H_{Y}$-periodic $B_{Y}$-module, and hence is isomorphic to $Y$. Hence, the $\mathbb{G}_{Y^{-}}$-orbit of the linear $H_{Y^{-}}$-periodic $\widetilde{B}_{Y^{-}}$ module $M_{Y}$ consists of representatives of the isoclasses of all weakly $\mathbb{G}_{Y}$-periodic $\widetilde{B}_{Y}$-modules. Therefore, by [DS3, Theorem 3.6], the tensor product functor

$$
-\otimes_{K\left[T, T^{-1}\right]} \mathbb{F}^{Y}\left(M_{Y}\right): \bmod K\left[T, T^{-1}\right] \longrightarrow \bmod B_{Y} / G_{Y},
$$

composed with the functor $\Pi^{Y}$, gives a natural $K$-linear equivalence of categories

$$
\bmod K\left[T, T^{-1}\right] \longrightarrow\left(\bmod B_{Y} / G_{Y}\right) /\left[\bmod _{1} B_{Y} / G_{Y}\right] .
$$

Observe also that $\mathbb{F}^{Y}\left(M_{Y}\right)=F^{Y}\left(F^{B_{Y}}\left(M_{Y}\right)\right)=F^{Y}(Y)$. Finally, it follows from [DS3, (2.4), (3.2)] that, for the tensor product functors

$$
\Psi^{Y}=-\otimes_{K\left[T, T^{-1}\right]} F^{Y}(Y): \bmod K\left[T, T^{-1}\right] \longrightarrow \bmod B_{Y} / G_{Y}
$$

and

$$
\Phi^{Y}=-\otimes_{K\left[T, T^{-1}\right]} F_{\lambda}(Y): \bmod K\left[T, T^{-1}\right] \longrightarrow \bmod A,
$$


the following diagram of functors is commutative:

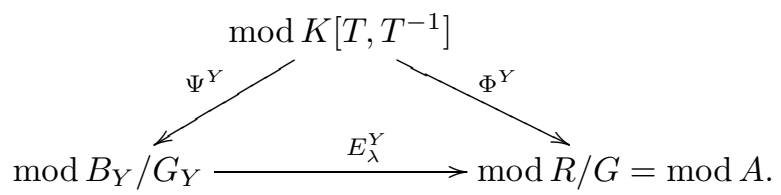

Therefore, the functor $\left(\Phi^{Y}\right): \coprod_{\mathcal{A}_{0}} \bmod K\left[T, T^{-1}\right] \rightarrow \bmod A$ composed with the functor $\Pi: \bmod A \rightarrow \bmod A /\left[\bmod _{1} A\right]$ gives a natural $K$-linear equivalence of categories

$$
\Phi: \coprod_{\mathcal{A}_{0}} \bmod K\left[T, T^{-1}\right] \longrightarrow \bmod A /\left[\bmod _{1} A\right]
$$

In particular, we conclude that every module $X$ in $\operatorname{ind}_{2} A$ is isomorphic to $\Phi^{Y}(V)=$ $V \otimes_{K\left[T, T^{-1}\right]} F_{\lambda}(Y)$ for some indecomposable finite dimensional $K\left[T, T^{-1}\right]$-module $V$. This proves statement (ii).

It follows from [DS3, Theorem 3.6] that the equivalences of categories $\bmod K[T$, $\left.T^{-1}\right] \rightarrow\left(\bmod B_{Y} / G_{Y}\right) /\left[\bmod _{1} B_{Y} / G_{Y}\right], Y \in \mathcal{A}_{0}$, established above, induce isomorphisms of translation quivers $\Gamma_{K\left[T, T^{-1}\right]} \cong\left(\Gamma_{B_{Y} / G_{Y}}\right)_{2}$, where $\left(\Gamma_{B_{Y} / G_{Y}}\right)_{2}$ is the translation subquiver of $\Gamma_{B_{Y} / G_{Y}}$ formed by all modules from $\operatorname{ind}_{2} B_{Y} / G_{Y}$. Therefore, the translation subquiver $\left(\Gamma_{A}\right)_{2}$ of $\Gamma_{A}$ formed by all modules from $\operatorname{ind}_{2} A$ is isomorphic to the disjoint union of $\left|\mathcal{A}_{0}\right|$ copies of the translation quiver $\Gamma_{K\left[T, T^{-1}\right]}$. Since the translation subquiver $\left(\Gamma_{A}\right)_{1}$ of $\Gamma_{A}$ formed by all modules from $\operatorname{ind}_{1} A$ is the orbit translation quiver $\Gamma_{R} / G$, the Auslander-Reiten quiver $\Gamma_{A}$ of $A$ has the required decomposition

$$
\Gamma_{A}=\left(\Gamma_{R} / G\right) \sqcup\left(\coprod_{\mathcal{A}_{0}} \Gamma_{K\left[T, T^{-1}\right]}\right) .
$$

This shows statement (iii).

For statement (i), observe that, since $R$ is cycle-finite, every finite convex subcategory of $R$ is of polynomial growth, by Theorem 2.2 Then applying [DS1, Lemma 3] we conclude that there exists a positive integer $m$ such that, for any dimension $d$, there exists a finite number of $K[T]$ - $A$-bimodules $M_{i}, 1 \leq i \leq n_{d} \leq d^{m}$, which are finitely generated and free as left $K[T]$-modules such that all but a finite number of isoclasses of modules in $\operatorname{ind}_{1} A$ of dimension $d$ are of the form $K[T] /(T-\lambda) \otimes_{K[T]} M_{i}$ for some $\lambda \in K$ and some $i \in\left\{1, \ldots, n_{d}\right\}$. This shows that the category $\bmod _{1} A$ of finite dimensional $A$-modules of the first kind is of polynomial growth, hence tame. Further, it follows from (ii) that every module $X$ in $\operatorname{ind}_{2} A$ is isomorphic to $K\left[T, T^{-1}\right] /(T-\mu)^{r} \otimes_{K\left[T, T^{-1}\right]} F_{\lambda}(Y)$ for some $Y \in \mathcal{A}_{0}=\mathcal{A}_{0}(R, G), \mu \in K \backslash\{0\}$ and $r \geq 1$. For each $Y \in \mathcal{A}_{0}$, consider the $K[S]$-A-bimodules

$$
Q_{Y, r}=K\left[S, T, T^{-1}\right] /(T-S)^{r} \otimes_{K\left[T, T^{-1}\right]} F_{\lambda}(Y), r \geq 1,
$$

which are clearly finitely generated and free as left $K[S]$-modules. Moreover, for every simple $K[S]$-module $K[S] /(S-\mu), \mu \in K \backslash\{0\}$, the (right) $A$-modules

$$
K[S] /(S-\mu) \otimes_{K[S]} Q_{Y, r} \text { and } K\left[T, T^{-1}\right] /(T-\mu)^{r} \otimes_{K\left[T, T^{-1}\right]} F_{\lambda}(Y)
$$


are isomorphic. Therefore, the category $\bmod _{2} A$ of finite dimensional $A$-modules of the second kind is tame. Summing up, we conclude that $A$ is tame, so (i) also holds.

We now exhibit properties of indecomposable locally finite dimensional modules over cycle-finite locally bounded categories established along the proof of Theorem 3.1 .

Corollary 3.2. Let $R$ be a connected cycle-finite locally bounded $K$-category, $Y$ a module in Ind $R$, and $G$ a torsion-free admissible group of $K$-linear automorphisms of $R$. Then $Y$ is a weakly $G$-periodic $R$-module if and only if the stabilizer $G_{Y}$ of $Y$ is nontrivial.

Proposition 3.3. Let $R$ be a connected cycle-finite locally bounded $K$-category, $G$ a torsion-free admissible group of $K$-linear automorphisms of $R$, and $Y$ a weakly $G$-periodic $R$-module. Then the following statements hold:

(i) $B_{Y}=\operatorname{supp} Y$ is an infinite convex subcategory of $R$ whose every finite convex subcategory is representation-finite.

(ii) $G_{B_{Y}}=G_{Y}$ is an infinite cyclic group.

(iii) There is a Galois covering $F^{B_{Y}}: \widetilde{B}_{Y} \rightarrow \widetilde{B}_{Y} / H_{Y}=B_{Y}$, where $\widetilde{B}_{Y}$ is a simply connected locally bounded $K$-category, whose every finite convex subcategory is representation-finite, and $B_{Y}=F^{B_{Y}}\left(L_{Y}\right)$ for an $H_{Y}$-periodic line $L_{Y}$ in $\widetilde{B}_{Y}$.

(iv) $Y \cong F_{\lambda}^{B_{Y}}\left(M_{L_{Y}}\right)$, where $F_{\lambda}^{B_{Y}}: \operatorname{Mod} \widetilde{B}_{Y} \rightarrow \operatorname{Mod} B_{Y}$ is the push-down functor associated to $F^{B_{Y}}$ and $M_{L_{Y}}$ is the canonical linear indecomposable $\widetilde{B}_{Y}$ module associated to the $H_{Y}$-periodic line $L_{Y}$.

(v) For a weakly $G$-periodic $R$-module $Z$ with $\operatorname{supp} Z=\operatorname{supp} Y$, we have $Y \cong$ $Z$.

As a direct consequence of Corollary 3.2 and [DS3, Proposition 2.3], described in Section [1, we obtain also the following result.

Corollary 3.4. Let $R$ be a connected cycle-finite locally bounded $K$-category and $G$ a torsion-free admissible group of $K$-linear automorphisms of $R$. Then $R$ is $G$-exhaustive if and only if $G$ acts freely on the isoclasses in Ind $R$.

It would be interesting to know if the $G$-exhaustivity of $R$, in the above corollary, forces $R$ to be locally support-finite, and consequently the equality Ind $R=$ ind $R$ (see [DS3, Proposition 2.5]).

The next result confirms Brenner's conjecture for the tame algebras having cyclefinite Galois coverings.

Theorem 3.5. Let $R$ be a connected cycle-finite locally bounded $K$-category, $G$ a torsion-free admissible group of $K$-linear automorphisms of $R$, and $A=R / G$. Let

$$
0 \longrightarrow \tau_{A} X \longrightarrow \bigoplus_{i=1}^{s(X)} Y_{i} \longrightarrow X \longrightarrow 0
$$


be an almost split sequence in $\bmod A$, where the modules $Y_{i}$ are indecomposable. Then $s(X) \leq 5$. Moreover, if $s(X)=5$, then one of the modules $Y_{i}$ is projectiveinjective.

Proof. This follows from Theorems 2.6 and 3.1, because the Auslander-Reiten quiver $\Gamma_{K\left[T, T^{-1}\right]}$ consists of a $K^{*}$-family of stable tubes of rank one.

We also mention that the assumption on the group $G$ to be torsion-free, posed in the above results, is justified by examples presented in GP.

\section{Criterion for polynomial Growth}

The aim of this section is to establish a criterion for an algebra with a cycle-finite Galois covering to be of polynomial growth (respectively, domestic type).

Theorem 4.1. Let $R$ be a connected cycle-finite locally bounded $K$-category, $G$ a torsion-free admissible group of $K$-linear automorphisms of $R$, and $A=R / G$. Then

(i) $A$ is of polynomial growth if and only if the number of $G$-orbits of isoclasses of weakly $G$-periodic $R$-modules is finite.

(ii) $A$ is domestic if and only if $R$ does not contain a convex subcategory which is tubular and the number of $G$-orbits of isoclasses of weakly G-periodic $R$-modules is finite.

Proof. Since every finite convex subcategory of $R$ is of polynomial growth, as a cycle-finite category, we know by [DS1, Lemma 3] that the category $\bmod _{1} A$ of finite dimensional $A$-modules of the first kind is of polynomial growth. Moreover, by Theorem 2.4 $\bmod _{1} A$ is domestic if and only if $R$ does not contain a finite convex subcategory which is a tubular algebra. We will discuss now the representation type of the category $\bmod _{2} A$ of finite dimensional $A$-modules of the second kind. We keep the notation from the proof of Theorem 3.1. It follows from Theorem 3.1 that every indecomposable module in $\bmod _{2} A$ is isomorphic to a module $\Phi^{Y}(V)=$ $V \otimes_{K\left[T, T^{-1}\right]} F_{\lambda}(Y)$ for some weakly $G$-periodic $R$-module $Y \in \mathcal{A}_{0}=\mathcal{A}_{0}(R, G)$ and some indecomposable finite dimensional $K\left[T, T^{-1}\right]$-module $V$. Hence, if $\mathcal{A}_{0}$ is finite, then the category $\bmod _{2} A$ is of domestic type. Therefore, if $\mathcal{A}_{0}$ is finite, then $A$ is of polynomial growth, and $A$ is domestic if and only if $R$ does not contain a finite convex subcategory which is a tubular algebra.

Assume that $\mathcal{A}_{0}$ is infinite. We will show that then the category $\bmod _{2} A$ is not of polynomial growth, and consequently $A$ is not of polynomial growth, and then not domestic. We will apply arguments similar to those used in the proof of Sk9, Proposition 1.2]. We know from Proposition 3.3 that, for each weakly $G$-periodic $R$-module $Y \in \mathcal{A}(R, G)$, its support $B_{Y}=\operatorname{supp} Y$ admits a simply connected Galois covering $F^{B_{Y}}: \widetilde{B}_{Y} \rightarrow \widetilde{B}_{Y} / H_{Y}=B_{Y}$ such that $B_{Y}=F^{B_{Y}}\left(L_{Y}\right)$ for a weakly $H_{Y^{-}}$ periodic line $L_{Y}$ in $\widetilde{B}_{Y}$, and $Y \cong F_{\lambda}^{B_{Y}}\left(M_{Y}\right)$ for the canonical linear $\widetilde{B}_{Y}$-module $M_{Y}=M_{L_{Y}}$ associated to $L_{Y}$. Moreover, every finite convex subcategory of $B_{Y}$ is a representation-finite standard algebra.

For each dimension $d$, denote by $\mu_{A}^{2}(d)$ the least number of $Y \in \mathcal{A}_{0}=\mathcal{A}_{0}(R, G)$ such that every module $X$ in $\operatorname{ind}_{2} A$ of dimension $d$ is isomorphic to $\Phi^{Y}(V)$ for some $Y \in \mathcal{A}_{0}$ and some indecomposable finite dimensional $K\left[T, T^{-1}\right]$-module $V$. 
Suppose that $\mu_{A}^{2}(d) \leq d^{m}$ for a fixed positive integer $m$ and all $d \geq 1$. Let $Y^{(n)}$, $n \geq 1$, be pairwise different modules from $\mathcal{A}_{0}$. Then it follows from Proposition 3.3 that the support categories $B^{(n)}=B_{Y^{(n)}}, n \geq 1$, are also pairwise different. Since $A=R / G$ is a finite category, there exists an object $x$ in $R$ whose $G$-orbit $G x$ intersects infinitely many categories $B^{(n)}, n \geq 1$. Without loss of generality, we may assume that $x$ belongs to all categories $B^{(n)}, n \geq 1$.

Observe that, for each $n \geq 1$, there are only finitely many categories $B_{Y}, Y \in$ $\mathcal{A}=\mathcal{A}(R, G)$, containing $x$ and with $B_{Y} / G_{Y}$ having at most $r$ objects. Further, if $R=K Q_{R} / I_{R}$, for an admissible ideal $I_{R}$ in $K Q_{R}$, then there is a common bound on the length of paths in $Q_{R}$ which do not belong to $I_{R}$, because $A=R / G$ is finite. This implies that there are two categories $B^{\prime}=B^{(i)}$ and $B^{\prime \prime}=B^{(j)}$ such that the intersection $B^{\prime} \cap B^{\prime \prime}$ contains the common category $F^{\prime}\left(L^{\prime}\right)=F^{\prime \prime}\left(L^{\prime \prime}\right)$, where $F^{\prime}=F^{B^{(i)}}, F^{\prime \prime}=F^{B^{(j)}}, L^{\prime}$ is a finite subline of the line $L_{Y^{(i)}}$ of $\widetilde{B}^{(i)}$ of the form

$$
a^{\prime} \longrightarrow y_{1}^{\prime} \longleftarrow \ldots \longleftarrow y_{p}^{\prime} \longleftarrow t^{\prime} \longrightarrow z_{s}^{\prime} \longrightarrow \ldots \longrightarrow z_{1}^{\prime} \longleftarrow b^{\prime}
$$

$L^{\prime \prime}$ is a finite subline of the line $L_{Y^{(j)}}$ of $\widetilde{B}^{(j)}$ of the form

$$
a^{\prime \prime} \longrightarrow y_{1}^{\prime \prime} \longleftarrow \ldots \longleftarrow y_{p}^{\prime \prime} \longleftarrow t^{\prime \prime} \longrightarrow z_{s}^{\prime \prime} \longrightarrow \ldots \longrightarrow z_{1}^{\prime \prime} \longleftarrow b^{\prime \prime}
$$

with $F^{\prime}\left(a^{\prime}\right)=a=F^{\prime \prime}\left(a^{\prime \prime}\right), F^{\prime}\left(y_{l}^{\prime}\right)=y_{l}=F^{\prime \prime}\left(y_{l}^{\prime \prime}\right)$, for $l \in\{1, \ldots, p\}, F^{\prime}\left(t^{\prime}\right)=t=$ $F^{\prime \prime}\left(t^{\prime \prime}\right), F^{\prime}\left(z_{k}^{\prime}\right)=z_{k}=F^{\prime \prime}\left(z_{k}^{\prime \prime}\right)$, for $k \in\{1, \ldots, s\}$, and $F^{\prime}\left(b^{\prime}\right)=b=F^{\prime \prime}\left(b^{\prime \prime}\right)$. Let $g \in G_{Y^{\prime}}$ be such that $g y_{1} \notin B^{\prime \prime}$ and $B^{\prime}$ contains a convex subcategory $C=F^{\prime}(v)$, for a subline $v$ of $L^{\prime}$ of the form

$$
y_{1}^{\prime} \longleftarrow \ldots \longleftarrow y_{p}^{\prime} \longleftarrow t^{\prime} \longrightarrow z_{s}^{\prime} \longrightarrow \ldots \longrightarrow z_{1}^{\prime} \longleftarrow \ldots \longrightarrow c_{1},
$$

with $F^{\prime}\left(c_{1}\right)=g y_{1}$. Similarly, let $h \in G_{Y^{\prime \prime}}$ be such that $h y_{1} \notin B^{\prime}$ and $B^{\prime \prime}$ contains a convex subcategory $D=F^{\prime \prime}(w)$ for a subline $w$ of $L^{\prime \prime}$ of the form

$$
y_{1}^{\prime \prime} \longleftarrow \ldots \longleftarrow y_{p}^{\prime \prime} \longleftarrow t^{\prime \prime} \longrightarrow z_{s}^{\prime \prime} \longrightarrow \ldots \longrightarrow z_{1}^{\prime \prime} \longleftarrow \ldots \longrightarrow d_{1},
$$

with $F^{\prime \prime}\left(d_{1}\right)=h y_{1}$. For each positive integer $n$, denote by $C^{n}$ the full subcategory of $B^{\prime}$ given by the objects of $C, g C, \ldots, g^{n-1} C$. Observe that $C^{n}=F^{\prime}\left(v^{n}\right)$, where $v^{n}$ is a convex connected subline of $L^{\prime}$ with ends $y_{1}^{\prime}$ and $c_{n}$ such that $F^{\prime}\left(c_{n}\right)=g^{n} y_{1}$. Similarly, for each positive integer $r$, denote by $D^{r}$ the full subcategory of $B^{\prime \prime}$ given by the objects of $D, h D, \ldots, h^{r-1} D$. Again, $D^{r}=F^{\prime \prime}\left(w^{r}\right)$, where $w^{r}$ is a convex connected subline of $L^{\prime \prime}$ with ends $y_{1}^{\prime \prime}$ and $d_{r}$ such that $F^{\prime \prime}\left(d_{r}\right)=h^{r} y_{1}$.

Let $M$ be the larger of the numbers of objects in $C$ and in $D$. Take a prime number $q$ such that

$$
2^{q}-2>2^{q-1}>q^{m+3} \text { and } q>M^{m+1} .
$$

For any sequence $(\mathbf{n}, \mathbf{r})=\left(n_{1}, r_{1}, n_{2}, r_{2}, \ldots, n_{s}, r_{s}\right)$ of integers $n_{i}, r_{i} \in \mathbb{N}$ with $s \geq 1$, $\sum_{i=1}^{t}\left(n_{i}+r_{i}\right)=q, \sum_{i=1}^{s} n_{i}>0, \sum_{i=1}^{s} r_{i}>0$, we denote by $E(\mathbf{n}, \mathbf{r})$ the full subcategory of $R$ given by the objects of

$$
C^{n_{1}}, g^{n_{1}} D^{r_{1}}, h^{r_{1}} g^{n_{1}} C^{n_{2}}, g^{n_{2}} h^{r_{1}} g^{n_{1}} D^{r_{2}}, \ldots, g^{n_{s}} h^{r_{s-1}} \ldots h^{r_{1}} g^{n_{1}} D^{r_{s}},
$$

and by $B_{f}$ the full subcategory of $R$ consisting of the objects $f^{i} x, i \in \mathbb{Z}, x \in E(\mathbf{n}, \mathbf{r})$, where $f=h^{r_{s}} g^{n_{s}} \ldots h^{r_{1}} g^{n_{1}}$. Observe that $B_{f}$ is a convex subcategory of $R$ whose every finite convex subcategory is a standard representation-finite algebra, admits 
a simply connected Galois covering $F^{(f)}: \widetilde{B}_{f} \rightarrow \widetilde{B}_{f} / H_{f}=B_{f}$, and $\widetilde{B}_{f}$ contains an $H_{f}$-periodic line $L_{f}$ such that $F^{(f)}\left(L_{f}\right)=B_{f}$. Further, the image $Y_{f}=F_{\lambda}^{(f)}\left(M_{f}\right)$ of the canonical linear indecomposable module $M_{f}=M_{L_{f}}$ associated to $L_{f}$ by the push-down functor $F_{\lambda}^{(f)}: \operatorname{MOD} \widetilde{B}_{f} \rightarrow$ MOD $B_{f}$ is a weakly $G$-periodic $R$-module with $B_{f}=\operatorname{supp} Y_{f}$. Moreover, the stabilizer $G_{Y_{f}}$ of $Y_{f}$ is the infinite cyclic group generated by $f$.

Let $(\mathbf{n}, \mathbf{r})=\left(n_{1}, r_{1}, \ldots, n_{s}, r_{s}\right)$ and $\left(\mathbf{n}^{\prime}, \mathbf{r}^{\prime}\right)=\left(n_{1}^{\prime}, r_{1}^{\prime}, \ldots, n_{t}^{\prime}, r_{t}^{\prime}\right)$ be two sequences of nonnegative integers satisfying the above condition, and $f=h^{r_{s}} g^{n_{s}} \ldots h^{r_{1}} g^{n_{1}}$, $f^{\prime}=h^{r_{t}^{\prime}} g^{n_{t}^{\prime}} \ldots h^{r_{1}^{\prime}} g^{n_{1}^{\prime}}$. Then the isoclasses of $Y_{f}$ and $Y_{f^{\prime}}$ belong to the same $G$-orbit if and only if one of the following two conditions holds:

(1) $\left(n_{1}^{\prime}, r_{1}^{\prime}, \ldots, n_{t}^{\prime}, r_{t}^{\prime}\right)=\left(b_{i}, r_{i}, n_{i+1}, r_{i+1}, \ldots, n_{s}, r_{s}, n_{1}, r_{1}, \ldots, n_{i-1}, r_{i-1}, c_{i}, 0\right)$ for some $i \geq 1$ and $b_{i}, c_{i} \in \mathbb{N}$ with $b_{i}+c_{i}=n_{i}$,

or

(2) $\left(n_{1}^{\prime}, r_{1}^{\prime}, \ldots, n_{t}^{\prime}, r_{t}^{\prime}\right)=\left(0, d_{i}, n_{i+1}, r_{i+1}, \ldots, n_{s}, r_{s}, n_{1}, r_{1}, \ldots, n_{i-1}, r_{i-1}, n_{i}, e_{i}\right)$ for some $i \geq 1$ and $d_{i}, e_{i} \in \mathbb{N}$ with $d_{i}+e_{i}=r_{i}$.

Therefore, there exist $\left(2^{q}-2\right) / q$ pairwise different $G$-orbits of isoclasses of weakly $G$-periodic $R$-modules of the form $Y_{f}$. For any element $f=h^{r_{s}} g^{n_{s}} \ldots h^{r_{1}} g^{n_{1}}$ associated to a sequence $(\mathbf{n}, \mathbf{r})=\left(n_{1}, r_{1}, \ldots, n_{s}, r_{s}\right)$, satisfying the imposed conditions, and an element $\lambda \in K^{*}=K \backslash\{0\}$, consider the $A$-module

$$
\mathbb{X}(\lambda, f)=K\left[T, T^{-1}\right] /(T-\lambda) \otimes_{K\left[T, T^{-1}\right]} F_{\lambda}\left(Y_{f}\right) .
$$

Then $\mathbb{X}(\lambda, f)$ is a module in $\operatorname{ind}_{2} A$ with $\operatorname{dim}_{K} \mathbb{X}(\lambda, f) \leq M q$. Moreover, $\mathbb{X}(\lambda, f) \cong$ $\mathbb{X}\left(\lambda^{\prime}, f^{\prime}\right)$ if and only if $\lambda=\lambda^{\prime}$ and the isoclasses of $Y_{f}$ and $Y_{f^{\prime}}$ belong to the same $G$-orbit. Therefore, we infer that

$$
\sum_{d \leq M q} \mu_{A}^{2}(d) \geq\left(2^{q}-2\right) / q
$$

Hence there exists $p \leq M q$ such that $\mu_{A}^{2}(p) \geq\left(2^{q}-2\right) /\left(M q^{2}\right)$. On the other hand, we have $\mu_{A}^{2}(p) \leq p^{m} \leq(M q)^{m}$ and so $2^{q}-2 \leq M^{m+1} q^{m+2}$. But, by our choice of $q$, we have $2^{q}-2>2^{q-1}>q^{m+3}>M^{m+1} q^{m+2}$, a contradiction. Therefore, if $\mathcal{A}_{0}=\mathcal{A}_{0}(R, G)$ is infinite, then $\bmod _{2} A$ is not of polynomial growth.

\section{EXAMPLES}

Here we exhibit some examples illustrating the main results of the paper.

5.1. Let $R$ be a locally bounded interval-finite $K$-category such that every finite convex subcategory of $R$ is of finite representation type, $G$ a torsion-free admissible group of $K$-linear automorphisms of $R$, and $A=R / G$. Then $R$ is a cycle-finite category and, applying Theorem 3.1, we conclude that $A$ is tame. We note that the special biserial algebras, playing the fundamental role in the representation theory, admit such Galois coverings (see [DS3], [ES], [PoS]).

5.2. A triangular locally bounded interval-finite $K$-category $R$ is called strongly simply connected if, for every finite convex subcategory $C$ of $A$, the first Hochschild 
cohomology space $H^{1}(C, C)$ vanishes (see $[\mathrm{Sk3}$ for other characterizations). It has been proved in [Sk8, Theorem 4.1] that a strongly simply connected algebra $\Lambda$ is of polynomial growth if and only if $\Lambda$ is cycle-finite. Therefore, every orbit algebra $A=R / G$ of a strongly simply connected locally bounded $K$-category $R$ of polynomial growth with respect to a torsion-free admissible group $G$ of $K$-linear automorphisms of $R$ is tame and admits a cycle-finite Galois covering (see also [Sk9, Theorem 2.4]).

5.3. We now exhibit an algebra $A$ which admits a cycle-finite Galois covering $F$ : $R \rightarrow R / G=A$ (with torsion-free group $G$ ) which is neither of type 5.1 nor of type 5.2 .

Let $A$ be the bound quiver algebra $K Q / I$ given by the quiver

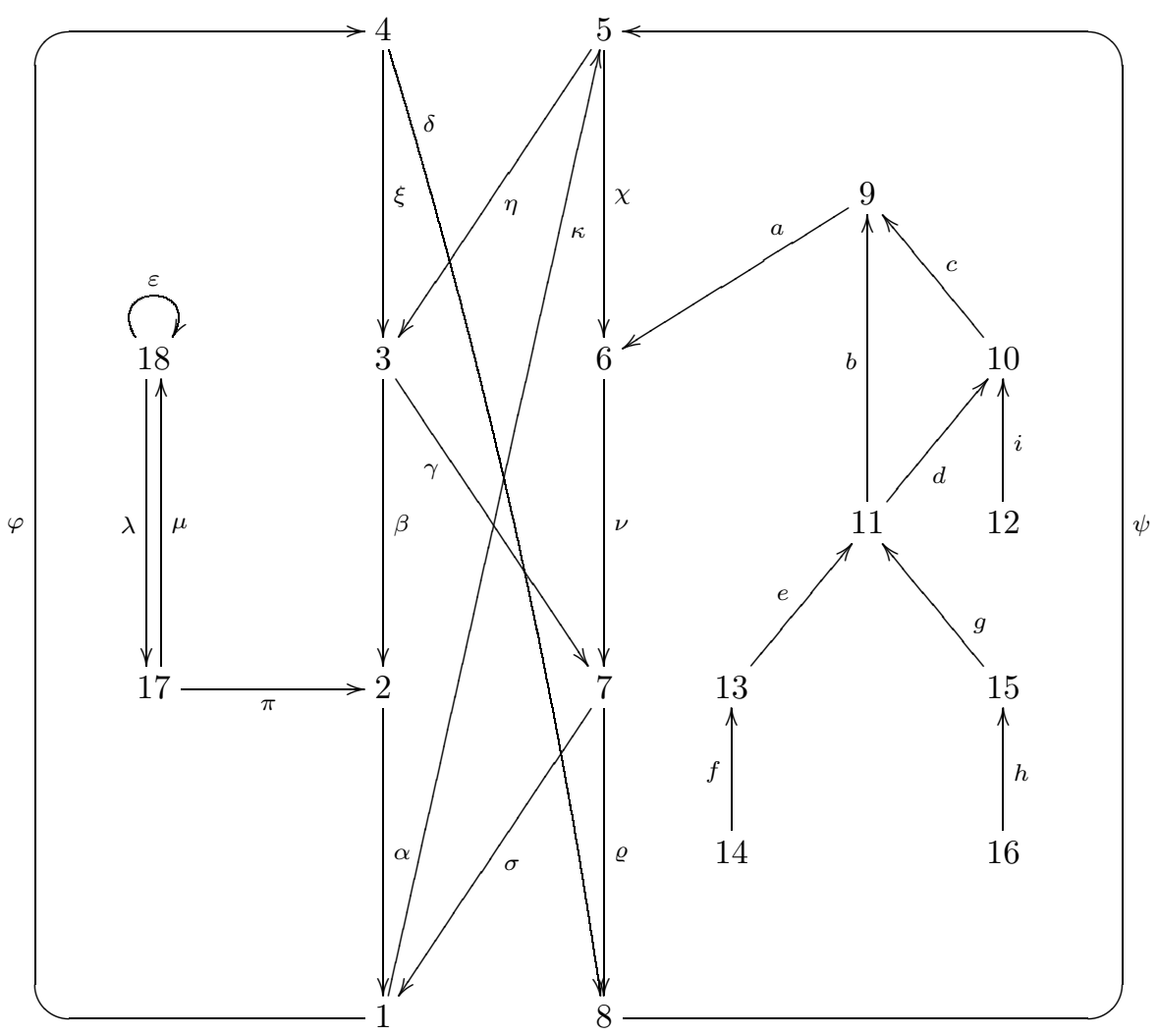

and the ideal $I$ of the path algebra $K Q$ of $Q$ generated by the elements $\alpha \varphi, \alpha \kappa$, $\sigma \varphi, \sigma \kappa, \gamma \sigma, \gamma \varrho, \nu \varrho, \nu \sigma, \eta \gamma, \eta \beta, \xi \beta, \xi \gamma, \varphi \delta, \delta \psi, \psi \eta, \psi \chi, \kappa \chi, \kappa \eta, a \nu, b a, d c a, i c, e b$, $g b-g d c, \pi \alpha, \lambda \pi, \mu \lambda \mu, \varepsilon^{2}-\lambda \mu, \mu \lambda-\mu \varepsilon \lambda$.

We note that the convex subcategory $B$ of $A$ given by the objects $9,10,11,12$, $13,14,15,16$ is a tubular algebra of tubular type $(3,3,3)$ which is simply connected (in the sense of AS2 ) but is not strongly simply connected, because it contains a 
convex hereditary subcategory of type $\widetilde{\mathbb{A}}_{3}$ given by the objects $9,10,11$. We then conclude that $A$ does not admit a strongly simply connected Galois covering.

For $K$ of characteristic 2, the convex subcategory $P$ of $A$ given by the objects 17 and 18 is a penny-farthing, and hence is a nonstandard representation-finite algebra. Hence, for $K$ of characteristic 2, the algebra $A$ does not admit a simply connected (even triangular) Galois covering.

The algebra $A$ admits a Galois covering $R \rightarrow R / G=A$, where $R$ is the bound quiver category $K \Delta / J$ with $\Delta$ the quiver of the form

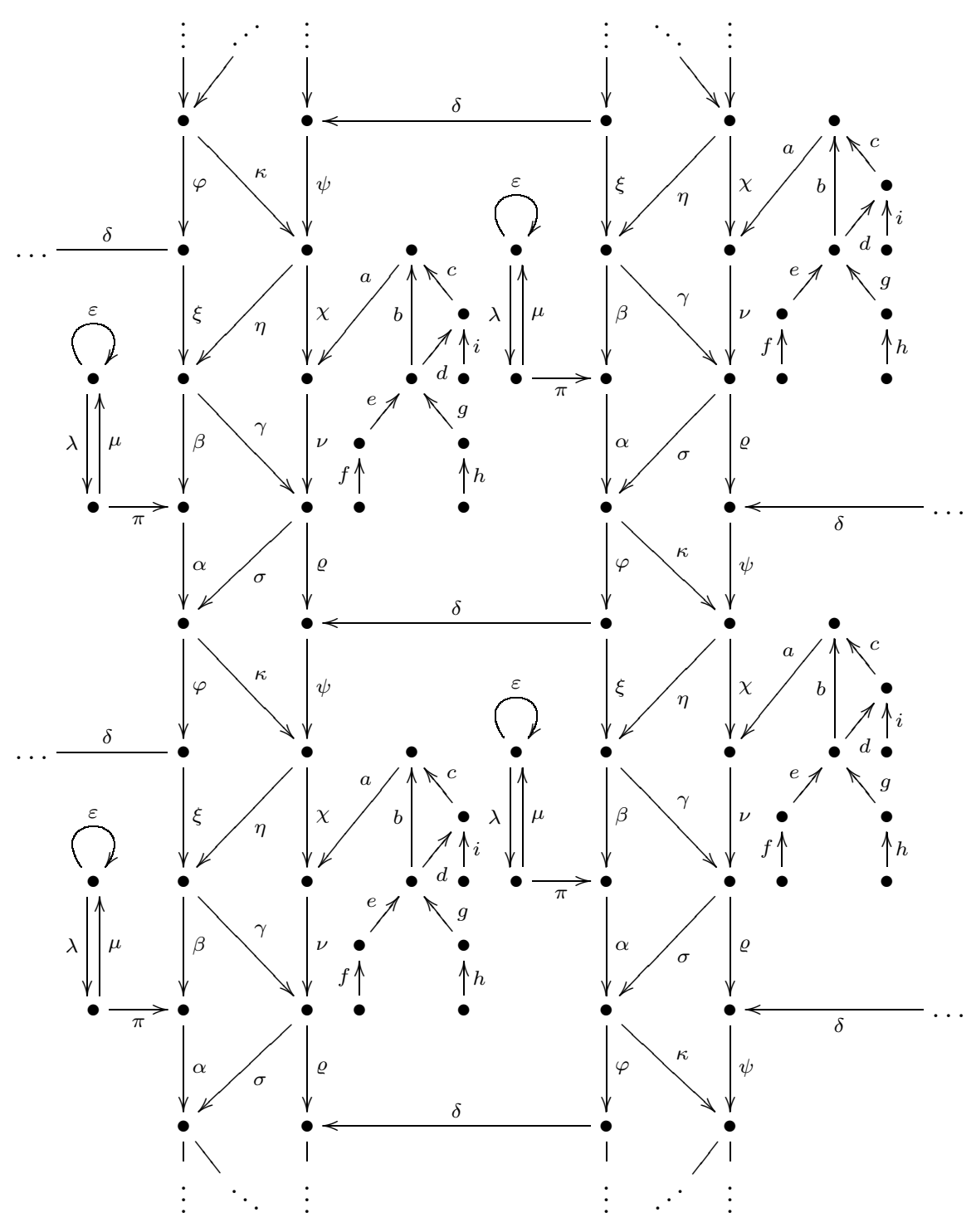


$J$ the ideal of the path category $K \Delta$ of $\Delta$ generated by all elements of the form $\alpha \varphi, \alpha \kappa, \sigma \varphi, \sigma \kappa, \gamma \sigma, \gamma \varrho, \nu \varrho, \nu \sigma, \eta \gamma, \eta \beta, \xi \beta, \xi \gamma, \varphi \delta, \delta \psi, \psi \eta, \psi \chi, \kappa \chi, \kappa \eta, a \nu, b a$, $d c a, i c, e b, g b-g d c, \pi \alpha, \lambda \pi, \mu \lambda \mu, \varepsilon^{2}-\lambda \mu, \mu \lambda-\mu \varepsilon \lambda$, and $G$ the free abelian group of rank 2 generated by the canonical horizontal and vertical shifts of $R=K \Delta / J$. Clearly, $R$ is a locally bounded interval-finite $K$-category. Applying the standard representation theory techniques one shows that the convex hull of the support of an indecomposable finite dimensional $R$-module $X$ is either a representation-finite subcategory containing one of the penny-farthings given by the arrows $\varepsilon, \lambda$, $\mu$, is contained in one of the tubular subcategories of tubular type $(3,3,3)$ given by the arrows $b, c, d, e, f, g, h, i$, or is a representation-finite subcategory of the special biserial subcategory of $R$ given by the subquiver of $\Delta$ consisting of all arrows except the arrows $\varepsilon, \lambda, \mu, b, d, e, f, g, h, i$. Therefore, $R$ is a cycle-finite locally bounded category, and hence, applying Theorem 3.1. we conclude that $A$ is a tame algebra. Further, applying Proposition 3.3, we conclude that the number of $G$-orbits of isoclasses of weakly $G$-periodic $R$-modules is infinite, and hence, by Theorem 4.1 , $A$ is not of polynomial growth. A typical weakly $G$-periodic $R$-module $Y$ is of the 
form

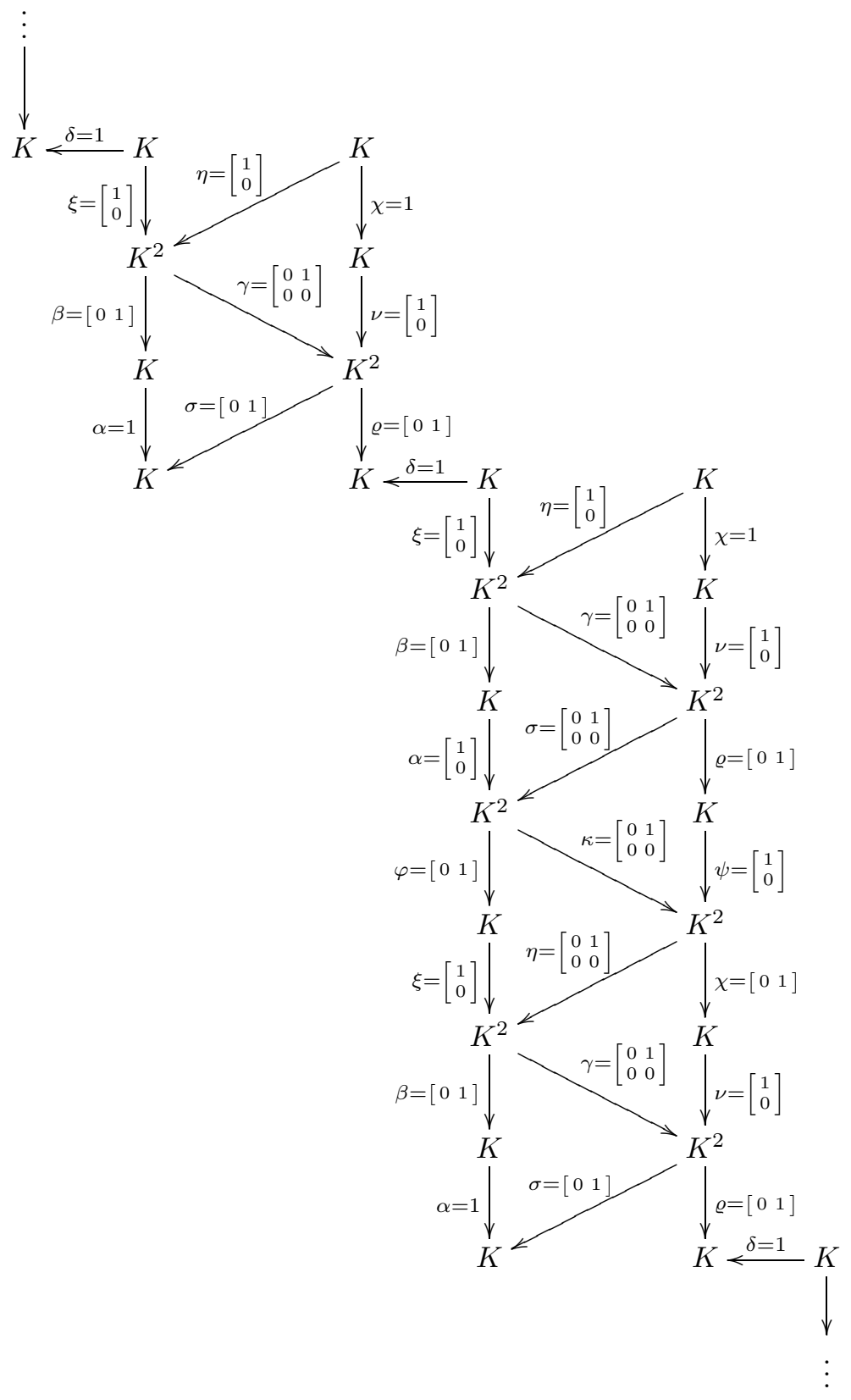

and the stabilizer $G_{Y}$ of $Y$ is the infinite cyclic subgroup of $G$ generated by the element $g \in G$ that shifts the linear map $K \ll^{\delta=1} K$ on the top to the linear map 
$K \gtrless^{\delta=1} K$ at the bottom. Then the $K\left[T, T^{-1}\right]$ - $A$-bimodule $F_{\lambda}(Y)$ is of the form

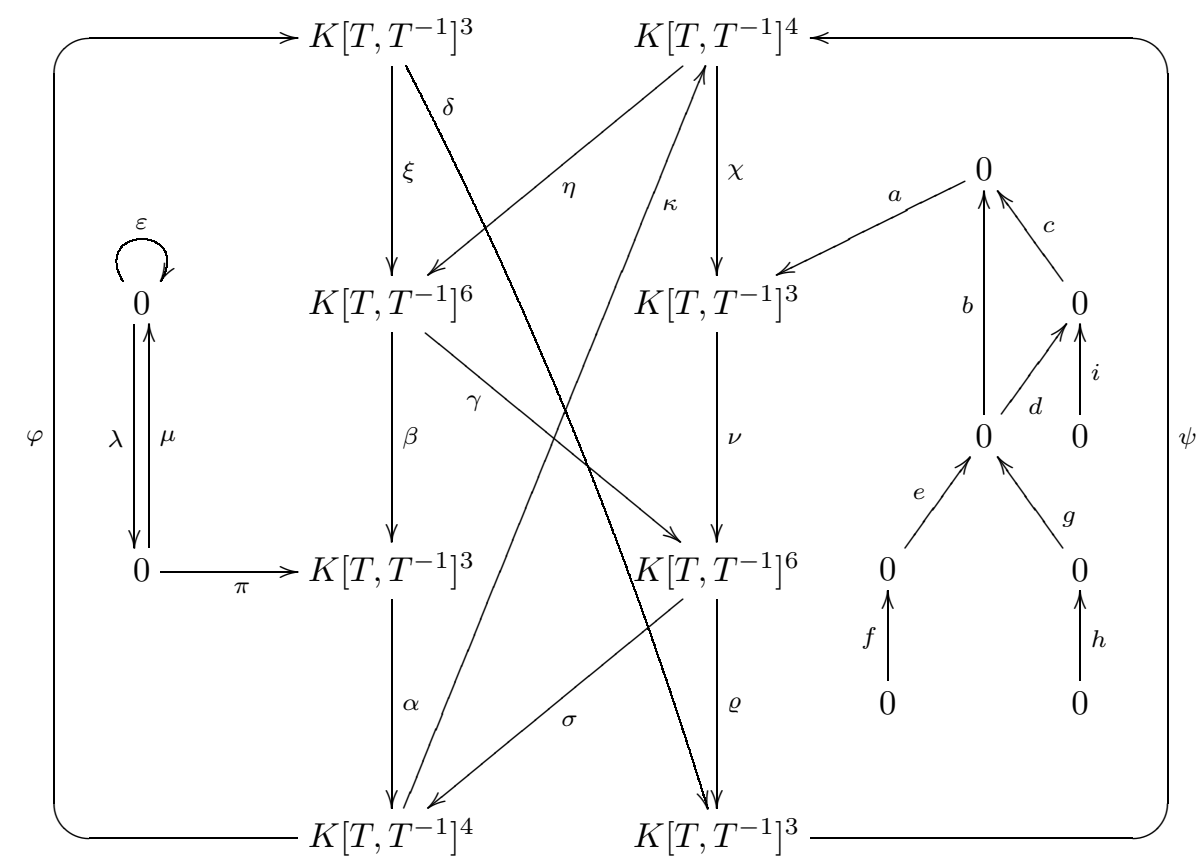

where $\xi, \eta, \gamma, \nu, \alpha, \sigma, \kappa, \psi, \beta, \varphi, \delta, \chi, \varrho$ are given by the matrices

$$
\begin{aligned}
& \xi=\left[\begin{array}{lll}
1 & 0 & 0 \\
0 & 0 & 0 \\
0 & 1 & 0 \\
0 & 0 & 0 \\
0 & 0 & 1 \\
0 & 0 & 0
\end{array}\right]=\nu, \eta=\left[\begin{array}{llll}
1 & 0 & 0 & 0 \\
0 & 0 & 0 & 0 \\
0 & 1 & 0 & 0 \\
0 & 0 & 0 & 0 \\
0 & 0 & 0 & 1 \\
0 & 0 & 0 & 0
\end{array}\right], \gamma=\left[\begin{array}{llllll}
0 & 1 & 0 & 0 & 0 & 0 \\
0 & 0 & 0 & 0 & 0 & 0 \\
0 & 0 & 0 & 1 & 0 & 0 \\
0 & 0 & 0 & 0 & 0 & 0 \\
0 & 0 & 0 & 0 & 0 & 1 \\
0 & 0 & 0 & 0 & 0 & 0
\end{array}\right], \\
& \alpha=\left[\begin{array}{llll}
1 & 0 & 0 & 0 \\
0 & 1 & 0 & 0 \\
0 & 0 & 0 & 0 \\
0 & 0 & 0 & 1
\end{array}\right], \sigma=\left[\begin{array}{llllll}
0 & 1 & 0 & 0 & 0 & 0 \\
0 & 0 & 0 & 1 & 0 & 0 \\
0 & 0 & 0 & 0 & 0 & 0 \\
0 & 0 & 0 & 0 & 0 & 1
\end{array}\right], \kappa=\left[\begin{array}{llll}
0 & 0 & 0 & 0 \\
0 & 0 & 0 & 0 \\
0 & 0 & 1 & 0 \\
0 & 0 & 0 & 0
\end{array}\right], \psi=\left[\begin{array}{lll}
0 & 0 & 0 \\
0 & 0 & 0 \\
0 & 1 & 0 \\
0 & 0 & 0
\end{array}\right] \\
& \beta=\left[\begin{array}{llllll}
0 & 1 & 0 & 0 & 0 & 0 \\
0 & 0 & 0 & 1 & 0 & 0 \\
0 & 0 & 0 & 0 & 0 & 1
\end{array}\right]=\varrho, \varphi=\left[\begin{array}{llll}
0 & 0 & 0 & 0 \\
0 & 0 & 0 & 0 \\
0 & 0 & 1 & 0
\end{array}\right], \delta=\left[\begin{array}{lll}
0 & 1 & 0 \\
0 & 0 & 0 \\
T & 0 & 0
\end{array}\right], \chi=\left[\begin{array}{llll}
1 & 0 & 0 & 0 \\
0 & 1 & 0 & 0 \\
0 & 0 & 0 & 1
\end{array}\right] \text {. }
\end{aligned}
$$

5.4. We now present an example of a tame algebra $A$ which admits a Galois covering $R \rightarrow R / G=A$ with infinite cyclic group $G$ and $R$ an interval-finite tame locally bounded $K$-category having infinitely many pairwise nonisomorphic weakly $G$-periodic modules with the support equal to $R$. This will show that the assumption on $R$ to be cycle-finite is necessary for the validity of Proposition 3.3 .

Let $A$ be the bound quiver algebra $K Q / I$ given by the quiver $Q$ of the form

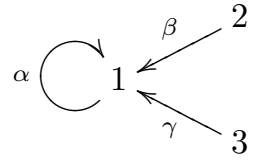


and the ideal $I$ of the path algebra $K Q$ generated by $\alpha^{2}$. The algebra $A$ admits a Galois covering $R \rightarrow R / G=A$, where $R$ is the bound quiver category $K \Delta / J$ with $\Delta$ the quiver of the form

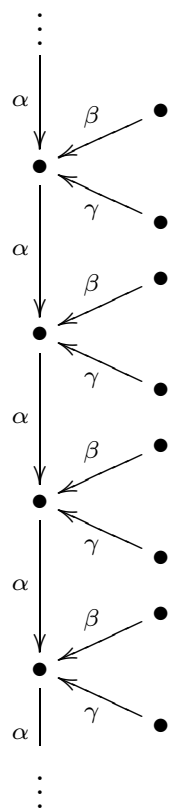

$J$ the ideal of the path category $K \Delta$ of $\Delta$ generated by all elements of the form $\alpha^{2}$, and $G$ is the infinite cyclic group generated by the canonical vertical shift of $R=K \Delta / J$.

Then $R$ is an interval-finite strongly simply connected locally bounded $K$-category which does not contain a hypercritical finite convex subcategory (see $[\mathbf{U}$ for the list of hypercritical algebras), and consequently $R$ is tame, by [BPS, Corollary 1]. On the other hand, $R$ contains the finite convex subcategory $R^{\prime}=K \Delta^{\prime} / J^{\prime}$ given by a subquiver $\Delta^{\prime}$ of $\Delta$ of the form

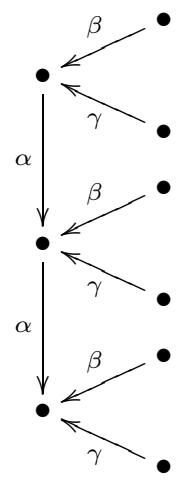

and the ideal $J^{\prime}$ of $K \Delta^{\prime}$ generated by $\alpha^{2}$, which is a $p g$-critical algebra in the sense of [NS. In particular, by [Sk8, Theorem 4.1], $R^{\prime}$ is not of polynomial growth, and hence is not cycle-finite. Therefore, $R$ is a tame strongly simply connected locally bounded $K$-category which is not cycle-finite. Moreover, it is not hard to show that there are infinitely many pairwise nonisomorphic weakly $G$-periodic $R$-modules with 
support equal to $R$. We also mention that $A$ is a tame algebra. For example, by [Bru, Lemma 5.3], $A$ degenerates (in the variety of $K$-algebras of dimension 8) to the special biserial bound quiver algebra $\bar{A}=K \bar{Q} / \bar{I}$ given by the quiver $\bar{Q}$ of the form

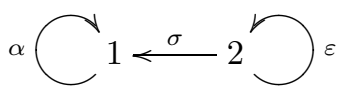

and the ideal $\bar{I}$ of the path algebra $K \bar{Q}$ generated by $\alpha^{2}$ and $\varepsilon^{2}$. Since $\bar{A}$ is tame (see [DS3, (5.2)]), $A$ is also tame, by [Ge] or [CB3].

\section{REFERENCES}

[ASS] I. Assem, D. Simson and A. Skowroński, Elements of the Representation Theory of Associative Algebras 1: Techniques of Representation Theory. London Mathematical Society Student Texts, Vol. 65, Cambridge University Press, Cambridge, 2006. MR2197389 (2006j:16020)

[AS1] I. Assem and A. Skowroński, Iterated tilted algebras of type $\widetilde{\mathbb{A}}_{n}$, Math. Z. 195 (1987), 269-290. MR892057 (88m:16033)

[AS2] I. Assem and A. Skowroński, On some classes of simply connected algebras, Proc. London Math. Soc. 56 (1988), 417-450. MR931509 (89f:16023a)

[AS3] I. Assem and A. Skowroński, Algebras with cycle-finite derived categories, Math. Ann. 280 (1988), 441-463. MR.936322 (89f:16023b)

[AS4] I. Assem and A. Skowroński, Minimal representation-infinite coil algebras, Manuscripta Math. 67 (1990), 305-331. MR1046991 (91h:16025)

[AS5] I. Assem and A. Skowroński, On tame repetitive algebras, Fund. Math. 142 (1993), 59-84. MR:1207471 (94a:16022)

[BB] R. Bautista and S. Brenner, On the number of terms in the middle of an almost split sequence, In: Representations of Algebras, Lecture Notes in Math., Vol. 903, SpringerVerlag, Berlin-Heidelberg, 1981, pp. 1-8. MR654699 (83f:16034)

[BGRS] R. Bautista, P. Gabriel, A. V. Roiter and L. Salmerón, Representation-finite algebras and multiplicative bases, Invent. Math. 81 (1985), 217-285. MR799266 (87g:16031)

[Bo1] K. Bongartz, Treue einfach zusammenhängende Algebren I, Comment. Math. Helv. 57 (1982), 282-330. MR684118 (84a:16051)

[Bo2] K. Bongartz, A criterion for finite representation type, Math. Ann. 269 (1984), 1-12. MR.756773 (86k:16023)

[Bo3] K. Bongartz, Critical simply connected algebras, Manuscripta Math. 46 (1984), 117-136. MR735517 (85j:16026)

[Bo4] K. Bongartz, Indecomposables are standard, Comment. Math. Helv. 60 (1985), 400-410. MR814147 (87d:16039)

[BoG] K. Bongartz and P. Gabriel, Covering spaces in representation theory, Invent. Math. 65 (1982), 337-378. MR643558 (84i:16030)

[BrG] O. Bretscher and P. Gabriel, The standard form of a representation-finite algebra, Bull. Soc. Math. France 111 (1983), 21-40. MR710374 (85g:16014)

[Bru] T. Brüstle, Tame tree algebras, J. Reine Angew. Math. 567 (2004), 51-98. MR2038305 (2004m:16014)

[BPS] T. Brüstle, J. A. de la Peña and A. Skowroński, Tame algebras and Tits quadratic forms, Advances Math. 226 (2011), 887-951.

[CB1] W. Crawley-Boevey, On tame algebras and bocses, Proc. London Math. Soc. 56 (1988), 451-483. MR 931510 (89c:16028)

[CB2] W. Crawley-Boevey, Tame algebras and generic modules, Proc. London Math. Soc. 63 (1991), 241-265. MR1114510 (92m:16019)

[CB3] W. Crawley-Boevey, Tameness of biserial algebras, Arch. Math. (Basel) 65 (1995), 399407. MR:1354686 (96i:16021)

[Do1] P. Dowbor, On the category of modules of second kind for Galois coverings, Fund. Math. 149 (1996), 31-54. MR:1372356 (97f:16028)

[Do2] P. Dowbor, Galois covering reduction to stabilizers, Bull. Polish Acad. Sci. Sér. Sci. Math. 44 (1996), 341-352. MR.1419407 (98a:16015) 
[Do3] P. Dowbor, Properties of G-atoms and full Galois covering reduction to stabilizers, Colloq. Math. 83 (2000), 231-265. MR1758318 (2001b:16014)

[Do4] P. Dowbor, Stabilizer conjecture for representation-tame Galois coverings of algebras, J. Algebra 239 (2001), 119-149. MR.1827877(2002e:16024)

[DS1] P. Dowbor and A. Skowroński, On Galois coverings of tame algebras, Arch. Math. (Basel) 44 (1985), 522-529. MR797444 (87a:16035)

[DS2] P. Dowbor and A. Skowroński, On the representation type of locally bounded categories, Tsukuba J. Math. 10 (1986), 63-77. MR846416 (88a:16057)

[DS3] P. Dowbor and A. Skowroński, Galois coverings of representation-infinite algebras, Comment. Math. Helv. 62 (1987), 311-337. MR896100 (88m:16020)

[Dre] P. Dräxler, Aufrichtige gerichtete Ausnahmealgebren, Bayreuth. Math. Schr. 29 (1989). MR.1007156 (90j:16045)

[Dro] Y. A. Drozd, Tame and wild matrix problems, In: Representation Theory II, Lecture Notes in Mathematics, Vol. 832, Springer-Verlag, Berlin-Heidelberg, 1980, pp. 242-258. MR 607157(83b:16024)

[ES] K. Erdmann and A. Skowroński, On Auslander-Reiten components of blocks and selfinjective biserial algebras, Trans. Amer. Math. Soc. 330 (1992), 165-189. MR.1144759 (93b:16022)

[Ga] P. Gabriel, The universal cover of a representation-finite algebra, In: Representations of Algebras, Lecture Notes in Math., Vol. 903, Springer-Verlag, Berlin-Heidelberg, 1981, pp. 68-105. MR654725 (83f:16036)

[Ge] C. Geiss, On degenerations of tame and wild algebras, Arch. Math. (Basel) 64 (1995), 11-16. MR.1305654 (95k:16014)

[GP] C. Geiss and J. A. de la Peña, An interesting family of algebras, Arch. Math. (Basel) 60 (1993), 25-35. MR.1193090 (94a:16023)

[HV] D. Happel and D. Vossieck, Minimal algebras of infinite representation type with preprojective component, Manuscripta Math. 42 (1983), 221-243. MR701205 (84m:16022)

[Ke] O. Kerner, Tilting wild algebras, J. London Math. Soc. 39 (1989), 29-47. MR989917 (90d:16025)

[LS] H. Lenzing and A. Skowroński, Quasi-tilted algebras of canonical type, Colloq. Math. 71 (1996), 161-181. MR:1414820 (97j:16019)

[Li] S. Liu, Almost split sequences for nonregular modules, Fund. Math. 143 (1993), 183-190. MR.1240634 (94g:16018)

[MPS] P. Malicki, J. A. de la Peña and A. Skowroński, Cycle-finite module categories, Preprint 2009.

[MS] P. Malicki and A. Skowroński, Algebras with separating almost cyclic coherent AuslanderReiten components, J. Algebra 291 (2005), 208-237. MR 2158519 (2006e:16034)

[NS] R. Nörenberg and A. Skowroński, Tame minimal non-polynomial growth simply connected algebras, Colloq. Math. 73 (1997), 301-330. MR.1446121 (98f:16012)

[MP] R. Martinez-Villa and J. A. de la Peña, The universal cover of a quiver with relations, J. Pure Appl. Algebra 30 (1983), 277-292. MR.724038 (85f:16035)

[PX] L. G. Peng and J. Xiao, On the number of DTr-orbits containing directing modules, Proc. Amer. Math. Soc. 118 (1993), 753-756. MR.1135078 (93i:16020)

[Pe1] J. A. de la Peña, Tame algebras with sincere directing modules, J. Algebra 161 (1993), 171-185. MR 1245849 (95b:16014)

[Pe2] J. A. de la Peña, The families of two-parametric tame algebras with sincere directing modules, In: Representations of Algebras, CMS Conf. Proc., Vol. 14, Amer. Math. Soc., Providence, RI, 1993, pp. 361-392. MR1265297 (95f:16013)

[PTa] J. A. de la Peña and M. Takane, On the number of terms in the middle of almost split sequences over tame algebras, Trans. Amer. Math. Soc. 351 (1999), 3857-3868. MR.1467463 (99m:16030)

[PTo] J. A. de la Peña and B. Tomé, Iterated tubular algebras, J. Pure Appl. Algebra 64 (1990), 303-314. MR 1061305 (91h:16028)

[Po] Z. Pogorzały, Regularly biserial algebras, In: Topics in Algebra, Banach Center Publ., Vol. 26, Part 1, PWN Warsaw, 1990, pp. 371-384. MR.1171245 (93m:16010)

[PoS $\quad$ Z. Pogorzały and A. Skowroński, Selfinjective biserial standard algebras, J. Algebra 138 (1991), 491-504. MR1102821 (92f:16012) 
[RS1] I. Reiten and A. Skowroński, Characterizations of algebras with small homological dimensions, Advances Math. 179 (2003), 122-154. MR2004730 (2004k:16029)

[RS2] I. Reiten and A. Skowroński, Generalized double tilted algebras, J. Math. Soc. Japan 56 (2004), 269-288. MR2027626(2005b:16039)

[Ri] C. M. Ringel, Tame Algebras and Integral Quadratic Forms, Lecture Notes in Mathematics, Vol. 1099, Springer-Verlag, Berlin-Heidelberg, 1984. MR774589 (87f:16027)

[SS1] D. Simson and A. Skowroński, Elements of the Representation Theory of Associative Algebras 2: Tubes and Concealed Algebras of Euclidean Type. London Mathematical Society Student Texts, Vol. 71, Cambridge University Press, Cambridge, 2007. MR 2360503 (2009f:16001)

[SS2] D. Simson and A. Skowroński, Elements of the Representation Theory of Associative Algebras 3: Representation-Infinite Tilted Algebras. London Mathematical Society Student Texts, Vol. 72, Cambridge University Press, Cambridge, 2007.

[Sk1] A. Skowroński, Selfinjective algebras of polynomial growth, Math. Ann. 285 (1989), 177199. MR 1016089 (90k:16024)

[Sk2] A. Skowroński, Algebras of polynomial growth, In: Topics in Algebra, Banach Center Publ., Vol. 26, Part 1, PWN Warsaw, 1990, pp. 535-568. MR.1171252 (93k:16026)

[Sk3] A. Skowroński, Simply connected algebras and Hochschild cohomologies, In: Representations of Algebras, CMS Conf. Proc., Vol. 14, Amer. Math. Soc., Providence, RI, 1993, pp. 431-437. MR1265301

[Sk4] A. Skowroński, Regular Auslander-Reiten components containing directing modules, Proc. Amer. Math. Soc. 120 (1994), 19-26. MR.1156473 (94b:16021)

[Sk5] A. Skowroński, Cycles in module categories, In: Finite Dimensional Algebras and Related Topics, NATO Adv. Sci. Int. Ser. C, Math. Phys. Sci., Vol. 424, Kluwer Acad. Publ., Dordrecht, 1994, pp. 309-345. MR.1308994 (96a:16010)

[Sk6] A. Skowroński, Cycle-finite algebras, J. Pure Appl. Algebra 103 (1995), 105-116. MR 1354071 (96i:16023)

[Sk7] A. Skowroński, Module categories over tame algebras, In: Representation Theory and Related Topics, Can. Math. Soc. Conf. Proc., Vol. 19, Amer. Math. Soc., 1996, pp. 281-313. MR 1388567 (97e:16033)

[Sk8] A. Skowroński, Simply connected algebras of polynomial growth, Compositio Math. 109 (1997), 99-133. MR1473607 (98k:16019)

[Sk9] A. Skowroński, Tame algebras with strongly simply connected Galois coverings, Colloq. Math. 72 (1997), 335-351. MR1426706 (97k:16016)

[Sk10] A. Skowroński, Tame quasi-tilted algebras, J. Algebra 203 (1998), 470-490. MR.1622799 (99b:16019)

[Sk11] A. Skowroński, Selfinjective algebras: Finite and tame type, In: Trends in Representation Theory of Algebras and Related Topics, Contemporary Mathematics, Vol. 406, Amer. Math. Soc., Providence, RI, 2006, pp. 169-238. MR2258046 (2007f:16045)

[U] L. Unger, The concealed algebras of the minimal wild, hereditary algebras, Bayreuther Math. Schriften 31 (1990), 145-154. MR1056151 (91i:16033)

Instituto de Matemáticas, UnAM, Ciudad Universitaria, 04510 México, D.F. México

E-mail address: jap@matem.unam.mx

Faculty of Mathematics and Computer Science, Nicolaus Copernicus University, Chopina 12/18, 87-100 Toruń, Poland

E-mail address: skowron@mat.uni.torun.pl 